Supporting Information

\title{
Bioactive Natural Small Molecule-Tuned Coassembly of Photosensitive Drugs for Highly Efficient Synergistic and Enhanced Type I Photochemotherapy
}

Jianjun Cheng ${ }^{\dagger}$, Haitian Zhao ${ }^{\dagger}$, Jiacheng Wang ${ }^{\dagger}$, Ying Han ${ }^{\dagger}$, Xin Yang ${ }^{*} \dot{ }$.

$\uparrow$ School of Chemistry and Chemical Engineering, Harbin Institute of Technology, No.92,

West Dazhi Street, Nangang District, Harbin, China 150001

E-mail: yangxin@hit.edu.cn

\section{Table of Contents}

Materials and Instruments

Methods S3-S10

Tables ..S11-S13

SEM images of free Ce6 assemblies .S14

SEM images of all NSMs-Ce6 co-assemblies

and free NSMs self-assemblies S14-S18

Additional Figures. S19-S37

References. S38-S39 


\section{Materials and Instruments}

Chlorin Ce6 was purchased from Jinan Daen Pharmaceutical Technology Co., Ltd (Shang Dong, China). Dimethyl sulfoxide (DMSO) and acetone were purchased from Sinopharm Chemical Reagent Co., Ltd (Shang Hai, China). Singlet oxygen fluorescence probe SOSG, Tween 80, ctDNA, 1,3-diphenylisobenzofuran (DPBF), 3-(4,5-dimethylthiazol-2-yl)-2,5dipheny-ltetrazolium bromide (MTT) were purchased from Sigma-Aldrich Chemical Company. Methylene blue (MB), 4,6-diamino-2-phenyl-indole (DAPI), Sodium azide, Dichlorofluorescin diacetate (DCFH-DA) were purchased from Alfa Aesar Chemical Company. Dihydroethidium (DHE) was obtained from Target Molecule Corp (Target Mol, China). Annexin V-FITC /PI, Calcein-AM/PI Double Stain Kit, RIPA lysis buffer (50 mM Tris-base, $150 \mathrm{mM} \mathrm{NaCl,0.1 \%}$ SDS, $1 \%$ Triton X-100, $0.5 \%$ sodium deoxycholate, $1 \mathrm{mM}$ sodium orthovanadate, $10 \mathrm{mM}$ sodium fluoride, $1 \mathrm{mM}$ phenylmethane-sulfonyl fluoride) were obtained from Beijing Slolarbio Science\& Technology Co., Ltd. All natural small molecules materials including tricyclic triterpenes: Poricoic acid (PAA); pentacyclic triterpenes: Betulinic acid (BA), Betulonic acid (BC), Glycyrrhetinic acid (GA), Ursolic acid (UA); Tricyclic diterpenes: Abietic acid (AA) were obtained according to our previous reported works. ${ }^{1,2}$ Tetracyclic triterpenes: Deoxycholic acid (DCA), Ursodeoxycholic acid (UDCA) and other tricyclic diterpenes: Abietic acid (AA), Dehydroabietic acid (DA), 12-Hydroxyabietic acid (12-HAA), 15-Hydroxydehydroabietic acid (15HDA) were purchased from Nanjing Spring \& Autumn Biological Engineering Co., Ltd (Nanjing, China). Fetal bovine serum (FBS), penicillin-streptomycin DMEM and RPMI 1640 Medium were purchased from Sigma Gibco (Grand Island, NY, USA). All reagents were used as received.

The mainly co-assembled reactions were performed on A KQ-100E ultrasonic cleaner. UV-vis absorption spectra were recorded at room temperature using TU-1900 PERSEE spectrophotometer. Fluorescence emission spectra were obtained on an F-2700 spectrometer. Fourier transform infrared (FT-IR) spectra were acquired using $\mathrm{KBr}$ disc samples in Nicolet iS5 (Thermo fisher, USA). Scanning electron transmission (SEM) images were recorded on aluminum foil plate using a Quanta 200FEG electron microscope operating at $20 \mathrm{Kv}$, and transmission electron microscope (TEM) images were recorded on Tecnai G2 F20 S-TWIN (FEI, America) operating at $200 \mathrm{kV}$, for which the sample aqueous dispersion was carefully placed onto micro-grid. The size distributions and Zeta potential were carried out with NanoBrook ZetaPALS Zeta Potential Analyzer (Brookhaven). And irradiation was performed using Solar-500 with a Xenon lamp (NBET, Beijing, China) with central wavelengths at $675 \pm 10 \mathrm{~nm}$. The cells were imaged using a fluorescence inverted microscope (UOP, DSY2000X), and the flow cytometry analysis was performed on a BD Accuri C6 Plus. A AllSHENG AMR-100 absorbance microplate reader was used in the MTT assay. 


\section{Methods}

\section{Preparation of NSMs-Ce6 NPs co-assemblies}

The NSMs mediated Ce6 co-assembled nanoparticles were prepared by one step reprecipitation method. Briefly, a stock DMSO solution of NSMs (33.54 mM) and Ce6 (33.54 mM) were first prepared. Then, $5 \mu \mathrm{L}$ of Ce 6 and $15 \mu \mathrm{L}$ of NSMs solutions were mixed by a Vortex mixer, and subsequently added rapidly into $1 \mathrm{~mL}$ DI water containing $10 \mu \mathrm{L} \mathrm{NaOH}(50.31 \mathrm{mM})$ solution under ultrasonication. After further sonication of the reaction mixture for $10 \mathrm{~min}$ at room temperature, the NSMs-Ce6 co-assemblies were obtained by centrifugation at $13000 \mathrm{rpm}$ for 25 min. Other formulations with different molar ratios of Ce6 to NSMs were also prepared by the same procedure with a constant $\mathrm{NaOH}$ to $\mathrm{Ce} 6$ molar ratio of 3:1. Except for the dissolution of betulonicacid (BC) in DMSO-acetone (Volume ratio 1: 1), all other natural small molecules tuned NSMs-Ce6 co-assemblies are prepared by dissolving the corresponding raw materials in DMSO.

\section{Drug loading efficiency in BC-Ce6 NPs}

The photosensitizer Ce6 and carrier betulonicacid (BC) loading efficiency in BC-Ce6 NPs were determined by HPLC (Agilent 1200 series liquid chromatography). For analytical, the obtained BC-Ce6 NPs were dissolved in DMSO and filtered through a Gelman Acrodisk PVDF membrane filter $(0.45 \mu \mathrm{m})$. The reversed phase TC-C18 column $(4.6 \mathrm{~mm}$ i.d. $\times 250 \mathrm{~mm}$, Agilent Technologies, USA) connected to an Agilent G1315B UV-Vis Detector was used at $30{ }^{\circ} \mathrm{C}$. The mobile phase was water $(0.2 \%$ phosphoric acid $)$ and acetonitrile at a flow rate of $1.0 \mathrm{~mL} / \mathrm{min}$, and corresponding volume ratios were 40:60 and 15:85 for $\mathrm{Ce} 6$ and $\mathrm{BC}$, respectively, while the detection wavelength was separately $402 \mathrm{~nm}$ and $212 \mathrm{~nm}$. Concentrations of Ce6 and BC were determined by a standard curve with a range of known concentrations. The drug loading efficiency (DLE) was calculated by the following equation: DLE $(\%)=$ [amount of Ce6 in the precipitate / (amount of Ce6 in the precipitate + amount of BC in the precipitate) $] \times 100 \%$.

\section{Reactive oxygen species (ROS) detection}

The ROS generation abilities of BC-Ce6 NPs was performed using a generally ROS probe 2,7-dichlorofluorescin diacetate (DCFH-DA), which could be oxidized to 2,7dichlorofluorescein (DCF) in the presence of $\operatorname{ROS}\left(\right.$ e.g. ${ }^{1} \mathrm{O}_{2}, \cdot \mathrm{OH}, \mathrm{O}_{2}{ }^{*}, \mathrm{H}_{2} \mathrm{O}_{2}$ ) after hydrolyzed to 2,7-dichlorodihydro fluorescein (DCFH), and then emitting strong fluorescence around 520 nm. ${ }^{3}$ Briefly, DCFH was prepared via a hydrolysis process of DCFH-DA under alkaline condition. Before detection, DCFH should be stored carefully at ice water without light. Then DCFH was added to Ce6 or BC-Ce6 NPs (equivalent Ce6: $2.5 \mu \mathrm{g} / \mathrm{mL}$ ) water solutions with a final concentrations of $30 \mu \mathrm{M}$. Afterward, the mixture was irradiated vigorously for various times by $675 \pm 10 \mathrm{~nm}$ light $\left(150 \mathrm{~mW} / \mathrm{cm}^{2}\right)$, and corresponding fluorescence emission spectra were measured with the excitation wavelength at $488 \mathrm{~nm}$. 


\section{Measurements of ${ }^{1} \mathrm{O}_{2}$ generation}

The ${ }^{1} \mathrm{O}_{2}$ generation abilities of BC-Ce6 NPs was performed by using a singlet oxygen fluorescence probe SOSG. ${ }^{4}$ Briefly, $1.5 \mu \mathrm{L}$ of SOSG $(2.5 \mathrm{mM})$ in methanol was added to free Ce6 or BC-Ce6 NPs (equivalent Ce6: $2 \mu \mathrm{g} / \mathrm{mL}$ ) water solutions, and then irradiated for various times. The fluorescence emission spectrum at different irradiation times was recorded with an excitation wavelength of $488 \mathrm{~nm}$. Meanwhile, BC-Ce6 NPs in water without SOSG probe were detected at the same conditions as a control group. In addition, $\mathrm{NaN}_{3}$, a specific quencher to ${ }^{1} \mathrm{O}_{2}$ production, was added prior to irradiation with a final concentration of $3.5 \mu \mathrm{M}$ for further confirmation of ${ }^{1} \mathrm{O}_{2}$ generation.

\section{Detection of ${ }^{1} \mathrm{O}_{2}$ quantum yield}

The ${ }^{1} \mathrm{O}_{2}$ quantum yield induced by BC-Ce6 NPs was employed by taking advantage of the 1,3-diphenylisobenzofuran (DPBF) decomposition reaction. Typically, $3 \mathrm{ml}$ of DPBF solution $\left(6 \times 10^{-5} \mathrm{~mol} / \mathrm{L}\right)$ was first transferred to a quartz cuvette $(1 \mathrm{~cm})$. After adding $20 \mu \mathrm{L}$ of photosensitizer $\mathrm{Ce} 6$ or BC-Ce6 NPs water solution (equivalent Ce6: $2.8 \times 10^{-5} \mathrm{~mol} / \mathrm{L}$ ), the mixture was irradiated and measured at $418 \mathrm{~nm}$ every $10 \mathrm{~s}$ for a $50 \mathrm{~s}$ period with a UV-Vis spectrophotometer. $3 \mathrm{ml}$ of DI water containing of $20 \mu \mathrm{L}$ sample was used to balance the instrument baseline. Methylene blue ( $\Phi=0.52)$ in $\mathrm{DMSO}^{5}$ was used as the reference compound. And the ${ }^{1} \mathrm{O}_{2}$ quantum yield $(\Phi)$ was calculated using the following equation:

$$
\Phi^{S}=\Phi^{R} \frac{m^{S} F^{R} P^{R}}{m^{R} F^{S} \bar{P}^{S}}
$$

Where $\Phi$ is ${ }^{1} \mathrm{O}_{2}$ quantum yield, Superscripts $S$ and $R$ separately represent the sample and reference compound, $m$ is a slope of a plot of the linear model, which was obtained by plotting absorption change $(\triangle \mathrm{OD})$ of DPBF at $418 \mathrm{~nm}$ as a function of irradiation time t. $F$ is the absorption correction factor at irradiation wavelength $\left(\mathrm{F}=1-10^{-\mathrm{OD}}\right)$. $\mathrm{P}$ is absorbed photonic flux.

\section{Measurements of $\mathrm{O}_{2} "$ generation}

The reaction between dihydroethidium (DHE) and DNA was used to evaluate the generation of superoxide anion $\left(\mathrm{O}_{2}{ }^{\circ}\right)$. In the presence of $\mathrm{O}_{2}{ }^{-{ }^{*}}$, DHE could be oxidized to ethidine, which can intercalate into DNA, emitting a bright fluorescence around $570 \mathrm{~nm} .{ }^{6}$ Briefly, $20 \mu \mathrm{L}$ of photosensitizer Ce6 or BC-Ce6 NPs water solutions (equivalent Ce6: $200 \mu \mathrm{g} / \mathrm{mL}$ ) and $10 \mu \mathrm{L}$ of DHE $(5 \mathrm{mM})$ in DMSO were dissolved in $2 \mathrm{~mL}$ of ctDNA $(200 \mu \mathrm{g} / \mathrm{mL})$ water solution. Then, the mixtures were irradiated by $675 \pm 10 \mathrm{~nm}$ light every $30 \mathrm{~s}$ for a $120 \mathrm{~s}$ period, and subsequently measured the fluorescence spectra with an excitation wavelength of $510 \mathrm{~nm}$. Meanwhile, a mixture only containing the DHE and ctDNA was used as a Control group.

\section{Measurements of $-\mathrm{OH}$ generation}

The $\cdot \mathrm{OH}$ generation was detected by $\cdot \mathrm{OH}$ assay Kit (Nangjing Jiancheng Bioengineering institute-HR Assay Kit, China). The $\cdot \mathrm{OH}$ could promote the occurrence of chromogenic reaction of Griess reagent forming a red azo dye (544 $\mathrm{nm}$ absorption). ${ }^{7}$ Typically, all reagents 
are prepared according to the Kit. Then, $0.2 \mathrm{~mL}$ of reaction substrate (reagent 2 of Kit) and 0.2 $\mathrm{mL}$ of photosensitizer Ce6 or BC-Ce6 NPs (equivalent Ce6: $2 \mu \mathrm{g} / \mathrm{mL}$ ) aqueous solution were mixed together. The mixtures were irradiated immediately by $675 \pm 10 \mathrm{~nm}$ light for various times when reagent 3 (Kit) was added. After reaction of $1 \mathrm{~min}$, the mixture was terminated rigorously with Griess reagent, and then measured the absorption of $544 \mathrm{~nm}$ by Uv-vis spectrometer. A free DI water $(0.2 \mathrm{~mL})$ instead of photosensitizer was added to the same system as the Control group.

\section{DFT calculation}

Molecular geometry optimizations of $\mathrm{Ce}$, betulonic acid (BC) and their aggregates were carried out through density functional theory (DFT) calculations with the Becke-3-Lee-YangParr (B3LYP) exchange function using the Gaussian 09 (Revision E.01) package. ${ }^{8}$ The 6-31G $(d, p)$ basis sets were used for all elements. And time-dependent DFT (TD-DFT) investigations were performed to explore the difference between monomer and their aggregates in both singlet and triplet excited states.

\section{Molecular Dynamics Simulation}

MD simulations were performed using the Materials Studio $8.0^{\mathrm{TM}}$ package. ${ }^{9}$ First, the system of Ce6 and BC molecules in a water box sized $4.5 \times 4.5 \times 4.5 \mathrm{~nm}^{3}$ were constructed and the density was set to $1.0 \mathrm{~g} \cdot \mathrm{cm}^{-3}$. Then, the system was optimized with 1000 steps of steepest descent minimization. After initial energy minimization and subsequently annealing at NVE ensemble ( $298 \mathrm{~K}-500 \mathrm{~K}$ ) for $20 \mathrm{ps}$, a total simulation time of $5.0 \mathrm{~ns}$ were performed in an NVT ensemble ( $298 \mathrm{~K}$ ) by Forcite model block. Berendsen thermostat was used, the time step was 1 fs, and the particle mesh Ewald method was used to calculate electrostatic interactions. The cutoff distance for nonbonded interaction was truncated at $12.5 \AA$. In all MD simulations, the COMPASS force field was used.

\section{In vitro drug release studies}

The in vitro release profiles were measured by a dialysis method. First, $1 \mathrm{~mL}$ of BC-Ce6 NPs (equivalent Ce6: $100 \mu \mathrm{g} / \mathrm{mL}$ ) PBS solutions ( $\mathrm{pH} 7.4$ or 6.5 ) containing $0.05 \%$ (v/v) Tween 80 were placed into dialysis bags $(\mathrm{Mw}$ cutoff $=7 \mathrm{kDa}$ ), and dialyzed in $100 \mathrm{~mL}$ of same PBS solution and shaken at $100 \mathrm{rpm}$ in a water bath at $37^{\circ} \mathrm{C}$. Then, $30 \mu \mathrm{L}$ of sample was collected from the dialysis bags at predetermined time points and subsequently dissolved in DMSO for determination of residual Ce6 by Uv-vis absorption spectroscopy. Meanwhile, $2 \mathrm{~mL}$ of PBS solutions outside the dialysis bag was removed and replenished with $2 \mathrm{~mL}$ of fresh PBS at each time point.

\section{Cell culture}

Human breast cancer cells (MCF-7), human liver hepatocellular cells (HepG2), mouse mammary cell line (4T1), and normal mouse fibroblast cell line (L929) were employed to evaluate the cell viabilities. MCF-7 and HepG2 cells were cultured in DMEM medium with 
$10 \%$ fetal bovine serum (FBS, Gibco) and $1 \%$ penicillin-streptomycin antibiotic (Life technology, USA). 4T1 and L929 were separately cultured in RMPI-1640 medium supplemented with $20 \%$ and $10 \%$ FBS containing $1 \%$ antibiotic at $37{ }^{\circ} \mathrm{C}$ under a $95 \%$ humidified atmosphere with $5 \% \mathrm{CO}_{2}$.

\section{Cellular uptake of BC-Ce6 NPs}

Intracellular uptake of BC-Ce6 NPs was observed by fluorescent inverted microscope (FIM) and analyzed using flow cytometry. Briefly, for FIM observation, 4T1 cells $\left(10^{5}\right)$ were seeded in 6-well plates and incubated for $24 \mathrm{~h}$ at $37^{\circ} \mathrm{C}$. Then the culture medium was replaced with 2 $\mathrm{mL}$ of free Ce6 or BC-Ce6 NPs (equivalent concentration of Ce6: $2 \mu \mathrm{g} / \mathrm{mL}$ ) fresh medium, and further incubated for $1 \mathrm{~h}$ or $4 \mathrm{~h}$, respectively. After fixing and DAPI staining of the cells, then imaged under FIM. Meanwhile, flow cytometric analysis was performed to evaluate the mean fluorescence intensity of cellular uptake. Same as above cell treatments, and cells treated without photosensitizer were set as control. Whereas the cells were successively harvested, centrifuged, washed with PBS, and re-suspended in $200 \mu \mathrm{L}$ of PBS, then $10^{4}$ cells were analyzed using an Accuri C6 flow cytometer (BD, Ann Arbor, MI).

\section{Cellular ROS generation}

A fluorescence probe DCFH-DA was used to evaluate the intercellular ROS generation level. In brief, 4T1 cells $\left(10^{5}\right)$ were seeded in 6-well plates. After incubation with free $\mathrm{Ce} 6$ or BCCe6 NPs (equivalent Ce6: $1.6 \mu \mathrm{g} / \mathrm{mL}$ ) for $4 \mathrm{~h}$, then $25 \mu \mathrm{M}$ DCFH-DA RMPI-1640 medium was added and incubated for another $30 \mathrm{~min}$. Whereafter, the cells were irradiated by light for $6 \mathrm{~min}$ and incubated for an additional $10 \mathrm{~min}$. After washing with PBS, the intercellular ROS generation was examined by FIM. The cells only incubated with DCFH-DA and irradiated were chosen as negative control.

\section{Cell Viability test}

The cytotoxicity of free natural small molecules (NSMs) and light activatable activity of free Ce6 or BC-Ce6 NPs were measured by performing standard MTT assay. For screening suitable nanocarrier, 4T1 cells $\left(1 \times 10^{4}\right)$ were seeded in 96 -well plate and incubated for $24 \mathrm{~h}$. The cells were then treated with a series of concentrations of free natural small molecules for $24 \mathrm{~h}$. Afterward, $10 \mu \mathrm{L}$ of MTT dye $(5 \mathrm{mg} / \mathrm{mL})$ was added to the well and incubated for additional 4 $\mathrm{h}$ before discarding the medium and dissolving the formazan crystals with $150 \mu \mathrm{L}$ DMSO. Finally, the absorbance of each well at $492 \mathrm{~nm}$ was recorded by a microplate reader.

The in vitro PDT activity of BC-Ce6 NPs was also measured by a similar MTT assay including cell numbers, detection process of MTT, and so on. Briefly, the experiments were mainly divided into two groups: 1) light group, the cells were treated with various equivalent concentrations of $\mathrm{Ce} 6$ or BC-Ce6 NPs for $4 \mathrm{~h}$, and irradiated for 10 min by $675 \pm 10 \mathrm{~nm}$ light $\left.\left(150 \mathrm{~mW} / \mathrm{cm}^{2}\right) ; 2\right)$ dark group, a plate similarly treated without irradiation was used as dark cytotoxicity group. After incubation for additional $20 \mathrm{~h}$, the cell viability was analyzed using MTT assay by comparing the absorbance at $492 \mathrm{~nm}$ of the control cells treated with fresh culture 
medium. Date are shown as the mean and standard error based on six independent experiments. In addition, the combination index (CI) analysis was calculated on the basis of the following equation:

$$
\mathrm{CI}=\frac{C_{A, X}}{I C_{X, A}}+\frac{C_{B, X}}{I C_{X, B}}
$$

Where $\mathrm{IC}_{X, A}$ and $\mathrm{IC}_{X, B}$ are the single drug concentrations when used alone to achieve $\mathrm{X} \%$ drug effect. $\mathrm{C}_{A, X}$ and $\mathrm{C}_{B, X}$ are separately represent the concentration of drug $\mathrm{A}$ and $\mathrm{B}$ when achieving the same effect in combination treatment. And $\mathrm{CI}$ is smaller than 1 indicating a synergistic therapeutic effects. ${ }^{[8]}$

\section{Type I and Type II mechanism of PDT}

In PDT, ROS such as ${ }^{1} \mathrm{O}_{2}$ (Type II reaction of PDT) and $-\mathrm{OH}$ (Type I reaction) play a vital role in the treatment of cancer. To further visualize the photochemical mechanism of BC-Ce6 mediated PDT in cell, sodium azide (SA) and D-mannitol (DM), a separately ${ }^{1} \mathrm{O}_{2}$ and $\cdot \mathrm{OH}$ specific quencher were employed to evaluate the PDT type. ${ }^{11}$ Precisely, 4 T1 cells $\left(2 \times 10^{4}\right)$ were seeded in 96-well plates and incubated for $24 \mathrm{~h}$. Then, the cells were treated in different ways including the following three groups: i) PDT group with various concentrations of BC-Ce6 NPs and irradiation; ii) PDT-SA group with BC-Ce6 NPs and irradiation, as well as $20 \mu \mathrm{L}$ SA (final concentration: $20 \mu \mathrm{M}$ ); iii) PDT-DM group with BC-Ce6 NPs and irradiation, as well as $20 \mu \mathrm{L}$ DM (final concentration: $40 \mu \mathrm{M}$ ). After incubation for $4 \mathrm{~h}$, the cells were exposed to light for $10 \mathrm{~min}$ and incubated for another $20 \mathrm{~h}$. The cell viability was also measured by MTT assay.

\section{Cell apoptosis assay}

For apoptosis analysis, Annexin V-FITC /PI (propidium Iodide) fluorescent dual staining was performed by flow cytometry. Briefly, 4T1 cells were seeded in 6-well plates and incubated with free Ce6 or BC-Ce6 NPs (Eq. Ce6: $2 \mu \mathrm{g} / \mathrm{mL}$ ) for $4 \mathrm{~h}$. After irradiation for $10 \mathrm{~min}$, the cells were incubated for additional $10 \mathrm{~h}$. Then, the cells were digested and washed according to the Kit guidelines. After staining with Annexin V-FITC/PI at room temperature for $5 \mathrm{~min}$ in the dark, the fluorescence of the cells was detected by the flow cytometry.

Besides, to further visualize the cytotoxicity of BC-Ce6 NPs PDT treatment, Calcein AM/propidium iodide (PI) live/dead staining was performed. Typically, 4T1 cells were seeded into $35 \mathrm{~mm}$ culture dishes $\left(2 \times 10^{5}\right.$ cells per well) and incubated until being $90 \%$ coverage. After incubation with $2 \mathrm{~mL}$ of free Ce6 or BC-Ce6 NPs (Eq. Ce6: $1.0 \mu \mathrm{g} / \mathrm{mL}$ ) for $4 \mathrm{~h}$, the cells were irradiated for $10 \mathrm{~min}$ and incubated for additional $16 \mathrm{~h}$. Then, washing the cells carefully with PBS for two times, and added $400 \mu \mathrm{L}$ Calcein AM-PI solutions for $20 \mathrm{~min}$. The final concentrations of AM and PI were 2 and $4.5 \mu \mathrm{M}$, respectively. Whereas, the cells were washed with PBS for 2 times and observed immediately under fluorescent inverted microscope with blue excitation for AM green fluorescence, and green excitation for PI red fluorescence. 


\section{Animal models}

Female Balb-c mice (18-22 g, 6-7 weeks old) were purchased from the Second Affiliated Hospital of Harbin Medical University. All animal experiments reported in this manuscript were performed under protocols approved by the laboratory animal research center of Harbin Medical University. 4T1 cells were collected and suspended In RMPI-1640 medium, and subcutaneously injected to the right-back of each mice.

\section{Hemolysis analysis}

Hemolysis analysis was used to evaluate the feasibility of intravenous administration and the potential biosafety of BC-Ce6 NPs. ${ }^{12}$ In brief, $10 \%$ (v/v) mouse blood red cells dispersed in cold PBS were first prepared. Then, $800 \mu \mathrm{L}$ of various concentrations of BC-Ce6 NPs PBS solutions were gently added to $200 \mu \mathrm{L}$ of red blood cells and incubated at room temperature for $4 \mathrm{~h}$. Afterward, the sample supernatants were collected by centrifugation at $13000 \mathrm{rpm}$ for 10 min, and immediately measured the absorbance at $577 \mathrm{~nm}$ using Uv-vis absorption spectra. Meanwhile, under the same conditions, $800 \mu \mathrm{L}$ of PBS solutions or distilled water without samples were added as a negative group and positive group, respectively. The Hemolytic percent of red blood cells can be calculated by the following equation:

$$
\text { Hemolytic percent (\%) }=\frac{A_{\text {Sample }}-A_{P B S}}{A_{\text {Water }}-A_{P B S}} \times 100 \%
$$

Where $\mathrm{A}_{\text {Sample }}, \mathrm{A}_{P B S}$ and $\mathrm{A}_{\text {water }}$ are the absorbance of each sample, PBS (negative control) and distilled water (positive control), respectively.

\section{Pharmacokinetics of BC-Ce6 NPs}

Healthy Balb-c mice were randomly divided into two groups $(\mathrm{n}=3)$ and intravenously (i.v.) injected $200 \mu \mathrm{L}$ of free Ce6 or BC-Ce6 NPs 5\% glucose solution (equivalent Ce6: $3.5 \mathrm{mg} / \mathrm{kg}$ ). At predetermined time intervals, $50 \mu \mathrm{L}$ of blood was withdrawn from posterior orbital venous plexus into heparinized tubes containing EDTA, and added to $50 \mu \mathrm{L}$ of cold blood lysis buffer (strong RIPA), then vortexed for 2 min and immediately lysed at $4{ }^{\circ} \mathrm{C}$ for $15 \mathrm{~min}$. After adding $250 \mu \mathrm{L}$ of DMSO to the mixture and further vortex for $2 \mathrm{~min}$, the Ce6 in blood supernatant was collected by centrifugation at $13000 \mathrm{rpm}$ for $10 \mathrm{~min}$. Finally, the concentrations of Ce6 were further analyzed by HPLC with a mobile phase of water ( $0.2 \%$ phosphoric acid) and acetonitrile (volume ratios, 40:60) as described above, and calculated according to a standard curve of known amounts of Ce6 in blood. Drug and statistics software (DAS ver 2.0) was used for the analysis of pharmacokinetic parameters.

\section{In/ex vivo fluorescence imaging}

For in vivo imaging of BC-Ce6 NPs, 4T1 cancer cells $\left(2.25 \times 10^{5}\right.$ cells per mouse) were subcutaneously injected to female Balb-c mice. When the tumor volumes approached $100 \mathrm{~mm}^{3}$, $200 \mu \mathrm{L}$ of BC-Ce6 NPs (equivalent Ce6: $0.3 \mathrm{mg} / \mathrm{mL}$ ) dispersed in $5 \%$ glucose solution were intravenously injected into the mice, then anesthetized and placed onto the warmed stage for imaging using AniView 100/600 Multi-model in vivo animal imaging system (Guangzhou 
Biolight Biotechnology Co., Ltd, China) at different time intervals with an exposure time of 1.0 s. The samples were excited at $630 \mathrm{~nm}$ and monitored at $680 \pm 10 \mathrm{~nm}$. After $24 \mathrm{~h}$ post-injection, the mice were sacrificed, and their tumors and major organs (heart, liver, spleen, lung, and kidney) were collected for ex vivo imaging, while the fluorescence intensity was quantified using the internal software of the instrument.

\section{In vivo antitumor evaluation of BC-Ce6 NPs}

To study the anticancer efficiency of BC-Ce6 NPs, $100 \mu \mathrm{L}$ of $4 \mathrm{~T} 1$ cancer cells were subcutaneously injected to female Balb-c mice at a density of $2.25 \times 10^{6}$ cells $/ \mathrm{mL}$. When the mean tumor volumes approached $75 \mathrm{~mm}^{3}$, the mice were randomly divided into five groups $(\mathrm{n}=5)$ and intravenously injected with $200 \mu \mathrm{L}$ of (i) $5 \%$ glucose aqueous solution, (ii) free betulonic acid (BC, $19.2 \mathrm{mg} / \mathrm{kg}$ ) (ii) free Ce6 with irradiation, (iv) BC-Ce6 NPs without irradiation or (v) BC-Ce6 NPs with irradiation at equivalent Ce6 of $4 \mathrm{mg} / \mathrm{kg}$ body. Where the free $\mathrm{BC}$ or $\mathrm{Ce} 6$ were first dissolved in ethanol-castor oil (volume ratio, 1: 1) and subsequently diluted with $5 \%$ glucose aqueous solution ensuring $5 \%$ (volume fraction) castor oil or ethanol, while BC-Ce6 NPs are dispersed in 5\% glucose aqueous solution. The mice were injected three times at 0th, 2th and 4th day, and the first injection day was designated as day 0. For irradiation groups, at $2 \mathrm{~h}$ post-injection after each administration, all mice were anesthetized with isoflurane, and the tumor site was irradiated by $675 \pm 10 \mathrm{~nm}$ light $\left(150 \mathrm{~mW} / \mathrm{cm}^{2}\right)$ for $15 \mathrm{~min}$. Then, the tumor size of mice were measured every two days using a digital caliper, and tumor volume was calculated by the formula: volume $=($ tumor length $) \times(\text { tumor width })^{2} \times 0.5$. Meanwhile, the body weight of mice was also measured every two days as indicators of systemic toxicity. After 14 days of treatment, all mice were sacrificed and their tumors were excised and weighted. The tumor inhibition ratio was calculated according to the equation:

$$
\text { Tumor inhibition ratio }(\%)=\left(1-\frac{m_{\text {sample }}}{m_{\text {control }}}\right) \times 100 \%
$$

Where $m_{\text {sample }}$ and $m_{\text {control }}$ were the average tumor weight of each treated group and $5 \%$ glucose aqueous solution group, respectively.

\section{Histological and TUNEL assays}

At the end of fourteen days PDT treatment, the mice were sacrificed, and the harvested tumors or major organs (heart, liver, spleen, lung, and kidney) were fixed with a $10 \%$ neutral buffered formalin and embedded in paraffin, then stained with haematoxylin and eosin (H\&E) for histological analysis. Meanwhile, terminal deoxynucleotidyl transferase-mediated deoxyuridine triphosphate nick-end labeling (TUNEL) assay was further performed to assess the tumor apoptosis levels in each groups using TUNEL kit (Roche Diagnostic, Indianapolis, IN, USA).

\section{Blood Chemistry}

Fourteen days after PDT treatments, the mice's blood were collected carefully and kept at $4{ }^{\circ} \mathrm{C}$ for $2 \mathrm{~h}$. After centrifugation at $3000 \mathrm{rpm}$ for $10 \mathrm{~min}$, the blood serum was obtained for 
biochemistry assay using an Automatic Biochemical Analyzer (Hitachi 7100, Japan). The recorded hematology data are mainly including liver function indexes: aspartate aminotransferase (AST), alanine aminotransferase (ALT), alkaline phosphatase (ALP); and renal function markers: creatinine (CREA), uric acid (UA), Urea (UREA); and other biochemical parameters: albumin (ALB), total bilirubin (TBIL).

\section{Statistical analysis}

All the data resulting from mumbles of independent experiments are expressed as the mean \pm standard deviation (mean \pm SD) unless otherwise noted. The two-sides student's test (SPSS 19.0 software, Chicago, IL, USA) was employed to compare the statistical significance. A probability values $<0.05$ was considered significant. Statistical significance: ${ }^{*} \mathrm{P}<0.05$, $* * \mathrm{P}<$ $0.01, * * * \mathrm{P}<0.001$. 


\section{Tables}

Table S1. Information and Morphology results of 17 natural small molecules (NSMs) mediated co-assemblies with Ce6.

\begin{tabular}{|c|c|c|c|c|c|c|}
\hline \multirow{2}{*}{$\begin{array}{l}\text { NSMs } \\
\text { (Types) }\end{array}$} & \multirow{2}{*}{ Name } & \multicolumn{4}{|c|}{ Morphology of NSMs-Ce6 co-assemblies ${ }^{\text {[a] }}$} & \multirow{2}{*}{$\begin{array}{l}\text { Diamet } \\
\text { er (nm) }\end{array}$} \\
\hline & & $1: 1$ & $1: 3$ & $1: 5$ & $1: 8$ & \\
\hline \multirow{7}{*}{$\begin{array}{l}\text { Pentacyclic } \\
\text { triterpenes }\end{array}$} & Betulinic acid (BA) & - & Fibrous & Fibrous & Fibrous & $\begin{array}{c}18.2^{[\mathrm{w}]} \\
(1: 5) \\
\end{array}$ \\
\hline & Betulin & - & - & - & - & \\
\hline & $\begin{array}{l}\text { Betulonic acid } \\
\text { (BC) }\end{array}$ & $\begin{array}{c}\text { Rod- } \\
\text { Spherical }\end{array}$ & Spherical & Spherical & Spherical & $\begin{array}{l}231.4 \\
(1: 3)\end{array}$ \\
\hline & $\begin{array}{l}\text { Glycyrrhetinic acid } \\
\text { (GA) }\end{array}$ & Spherical & Lamellar & Net-like & Rod-like & $\begin{array}{l}175.5 \\
(1: 1)\end{array}$ \\
\hline & Ursolic acid (UA) & Clavate & Clavate & Clavate & Clavate & $\begin{array}{l}197.8 \\
(1: 3) \\
\end{array}$ \\
\hline & Glycyrrhizic acid & - & - & - & - & \\
\hline & Echinocystic acid & - & - & - & - & \\
\hline \multirow{5}{*}{$\begin{array}{l}\text { Tetracyclic } \\
\text { triterpenes }\end{array}$} & Cholic acid & - & - & - & - & \\
\hline & $\begin{array}{c}\text { Deoxycholic acid } \\
\text { (DCA) }\end{array}$ & - & Spherical & - & - & $\begin{array}{l}270.5 \\
(1: 3)\end{array}$ \\
\hline & $\begin{array}{c}\text { Hyodeoxycholic } \\
\text { acid }\end{array}$ & - & - & - & - & \\
\hline & $\begin{array}{c}\text { Ursodeoxycholic } \\
\text { acid (UDCA) }\end{array}$ & - & $\begin{array}{c}\text { Rod- } \\
\text { Spherical } \\
\end{array}$ & $\begin{array}{l}\text { Spherical } \\
\text {-pleated }\end{array}$ & - & $\begin{array}{l}334.7 \\
(1: 3) \\
\end{array}$ \\
\hline & $\begin{array}{c}\text { Chenodeoxycholic } \\
\text { acid }\end{array}$ & - & - & - & - & \\
\hline $\begin{array}{l}\text { Tricyclic } \\
\text { triterpenes }\end{array}$ & $\begin{array}{c}\text { Poricoic acid } \\
\text { (PAA) }\end{array}$ & - & Fiber-rod & Fibrous & Net -like & $\begin{array}{l}376.5 \\
(1: 3)\end{array}$ \\
\hline \multirow{4}{*}{$\begin{array}{l}\text { Tricyclic } \\
\text { diterpenes }\end{array}$} & $\begin{array}{l}\text { Dehydroabietic } \\
\text { acid (DA) }\end{array}$ & Flake & Rod-like & Spherical & Spherical & $\begin{array}{l}193.8 \\
(1: 8)\end{array}$ \\
\hline & $\begin{array}{c}\text { 15-Hydroxy } \\
\text { dehydroabietic acid } \\
\text { (15HDA) }\end{array}$ & - & Spherical & - & Rod-like & $\begin{array}{l}212.1 \\
(1: 3)\end{array}$ \\
\hline & Abietic acid (AA) & Spherical & Spherical & - & - & $\begin{array}{l}178.7 \\
(1: 3) \\
\end{array}$ \\
\hline & $\begin{array}{c}\text { 12-Hydroxyabietic } \\
\text { acid (12HAA) }\end{array}$ & - & Spherical & - & Spherical & $\begin{array}{l}153.9 \\
(1: 3)\end{array}$ \\
\hline
\end{tabular}

[a] different molar ratios of Ce6 to free NSMs. [b] the average DLS diameter of NSMs-Ce6 NPs at the specified molar ratio. [w] the width of fibrous NPs obtained from SEM. "_" Amorphous morphology. 
Table S2. The $\mathrm{IC}_{50}$ analysis and detailed information on free identified natural small molecules.

\begin{tabular}{|c|c|c|c|}
\hline \multicolumn{2}{|c|}{ Natural small molecules } & \multicolumn{2}{|c|}{$\mathrm{IC}_{50}$ for $4 \mathrm{~T} 1$ cells } \\
\hline Types & Free materials & $\begin{array}{l}\text { Mean } \pm \text { SD } \\
(\mu \mathrm{M})\end{array}$ & $\begin{array}{c}\text { Mean value } \\
(\mu \mathrm{g} / \mathrm{mL})\end{array}$ \\
\hline \multirow{4}{*}{$\begin{array}{l}\text { Tricyclic } \\
\text { diterpenes }\end{array}$} & Dehydroabietic acid (DA) & $571.27 \pm 26.70$ & 171.63 \\
\hline & $\begin{array}{c}\text { 15-Hydroxy } \\
\text { dehydroabietic acid } \\
\text { (15HDA) }\end{array}$ & $2487.29 \pm 87.85$ & 787.05 \\
\hline & $\begin{array}{l}\text { 12-Hydroxyabietic acid } \\
\text { (12HAA) }\end{array}$ & $1428.20 \pm 67.23$ & 454.81 \\
\hline & Abietic acid (AA) & $281.42 \pm 12.08$ & 85.11 \\
\hline $\begin{array}{l}\text { Tricyclic } \\
\text { triterpenes }\end{array}$ & Poricoic acid (PAA) & $235.46 \pm 17.83$ & 124.50 \\
\hline \multirow{4}{*}{$\begin{array}{l}\text { Pentacyclic } \\
\text { triterpenes }\end{array}$} & Betulinic acid (BA) & $19.74 \pm 0.50$ & 9.02 \\
\hline & Betulonic acid (BC) & $58.62 \pm 4.50$ & 26.65 \\
\hline & Ursolic acid (UA) & $40.05 \pm 3.74$ & 18.29 \\
\hline & Glycyrrhetinic acid (GA) & $1511.92 \pm 63.75$ & 711.63 \\
\hline \multirow{2}{*}{$\begin{array}{l}\text { Tetracyclic } \\
\text { triterpenes }\end{array}$} & $\begin{array}{l}\text { Ursodeoxycholic acid } \\
\text { (UDCA) }\end{array}$ & --- & --- \\
\hline & Deoxycholic acid (DCA) & --- & --- \\
\hline
\end{tabular}

Table S3. The UV absorption wavelength at Soret and Qy bands of BC-Ce6 NPs prepared at different initial molar ratios of $\mathrm{Ce} 6$ to $\mathrm{BC}$, respectively.

\begin{tabular}{ccccccc}
\hline Formulations $^{[\mathrm{a}]}$ & $\mathrm{Ce} 6$ & $2: 1$ & $1: 1$ & $1: 3$ & $1: 5$ & $1: 8$ \\
\hline $\begin{array}{c}\text { Soret bands } \\
(\mathrm{nm})\end{array}$ & 404 & 402 & 408 & 409 & 409 & 410 \\
$\begin{array}{c}\mathrm{Qy} / \lambda \max \\
(\mathrm{nm})\end{array}$ & 665 & 659 & 667 & 669 & 669 & 670 \\
\hline
\end{tabular}

[a] different initial molar ratio of $\mathrm{Ce} 6$ to $\mathrm{BC}$ 
Table S4. The Combination Index (CI) analysis of $\mathrm{BC} / \mathrm{Ce} 6$ mixture with irradiation when achieving $25 \%, 50 \%, 75 \%$ drug effect. Where the concentration ratio of Ce6 to $\mathrm{BC}(\mu \mathrm{g} / \mathrm{mL})$ in $\mathrm{BC}+\mathrm{Ce} 6$ mixture is $1: 17.2$.

\begin{tabular}{cccccc}
\hline Formulations & BC & $\begin{array}{c}\text { BC in } \\
\text { mixture }\end{array}$ & Ce6 & $\begin{array}{c}\text { Ce6 in } \\
\text { mixture }\end{array}$ & CI \\
\hline $\mathrm{IC}_{25}$ & $19.365 \pm 1.21$ & $7.213 \pm 0.13$ & $0.735 \pm 0.12$ & $0.359 \pm 0.04$ & 0.86 \\
$\mathrm{IC}_{50}$ & $41.250 \pm 1.42$ & $12.011 \pm 0.38$ & $1.362 \pm 0.13$ & $0.638 \pm 0.04$ & 0.75 \\
$\mathrm{IC}_{75}$ & $87.869 \pm 3.37$ & $19.844 \pm 0.69$ & $2.521 \pm 0.33$ & $1.163 \pm 0.01$ & 0.68 \\
\hline
\end{tabular}

Table S5. The IC 50 value of BC-Ce6 NPs on tumor cells including 4T1, MCF-7, HepG2, and normal cell L929, respectively.

\begin{tabular}{ccccc}
\hline \multirow{2}{*}{ IC $_{50}$} & \multicolumn{3}{c}{ Tumour cells } & Normal cell \\
\cline { 2 - 5 } & 4T1 & MCF-7 & HepG2 & L929 \\
\hline $\begin{array}{c}\text { BC-Ce6 NPs } \\
(\text { Eq. Ce6 } \mu \mathrm{g} / \mathrm{mL})\end{array}$ & $0.30 \pm 0.02$ & $0.48 \pm 0.05$ & $0.41 \pm 0.03$ & $10.36 \pm 0.84$ \\
\hline
\end{tabular}

Table S6. Pharmacokinetic parameters of Ce6 after intravenous administration of BC-Ce6 NPs with equivalent Ce6 concentrations of $3.5 \mathrm{mg} / \mathrm{kg}$ in rats $(\mathrm{n}=3)$.

\begin{tabular}{ccccccc}
\hline Parameters & $\begin{array}{c}\mathrm{AUC}(0-\infty) \\
(\mu \mathrm{g} / \mathrm{mL} \cdot \mathrm{h})\end{array}$ & $\begin{array}{c}\mathrm{AUC}(0-\mathrm{t}) \\
(\mu \mathrm{g} / \mathrm{mL} \cdot \mathrm{h})\end{array}$ & $\begin{array}{c}\mathrm{CL} \\
(\mathrm{L} / \mathrm{h})\end{array}$ & $\begin{array}{c}\mathrm{MRT}(0-\infty) \\
(\mu \mathrm{g} / \mathrm{mL} \cdot \mathrm{h})\end{array}$ & $\begin{array}{c}\mathrm{T}(1 / 2) \\
(\mathrm{h})\end{array}$ & $\begin{array}{c}\mathrm{Cmax} \\
(\mu \mathrm{g} / \mathrm{mL})\end{array}$ \\
\hline Ce6 & $5.855 \pm 0.968$ & $4.549 \pm 0.752$ & 0.012 & $1.288 \pm 0.213$ & $0.958 \pm 0.158$ & $5.502 \pm 0.910$ \\
$\begin{array}{c}\text { BC-Ce6 } \\
\text { NPs }\end{array}$ & $63.364 \pm 3.385$ & $57.762 \pm 1.064$ & 0.001 & $5.693 \pm 0.286$ & $3.476 \pm 0.526$ & $23.829 \pm 2.246$ \\
\hline
\end{tabular}



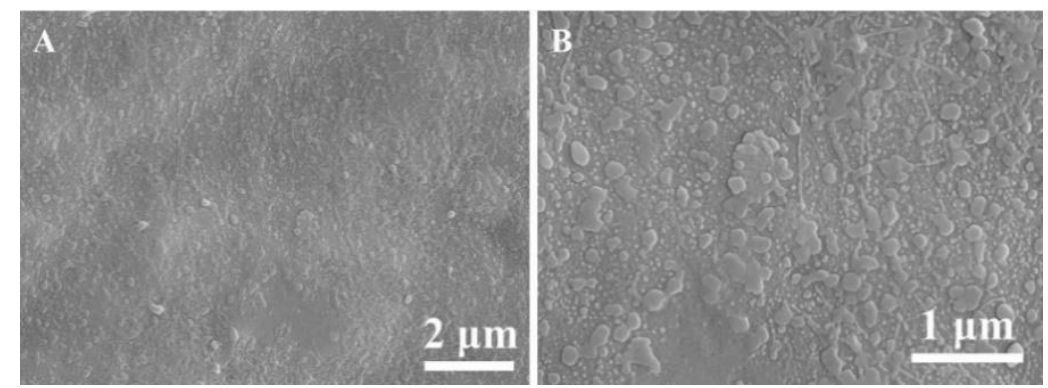

Figure S1. SEM images at (A) magnify $\times 20 \mathrm{~K}$ and $(\mathrm{B}) \times 50 \mathrm{~K}$ of free $\mathrm{Ce} 6$ assemblies, respectively. Where free Ce6 assemblies were prepared via same reprecipitation method for preparation of NSMs-Ce6 co-assemblies. After centrifugation of the reaction solutions, a large number of free NSMs self-assemblies could be observed, but only a few Ce6 precipitates were obtained for SEM imaging.

\section{SEM images of all NSMs-Ce6 co-assemblies and free NSMs self-assemblies}

Figure S2. SEM images (as follows) of the identified 11 NSMs mediated NSMs-Ce6 coassemblies (e.g., BC-Ce6 co-assemblies, AA-Ce6 co-assemblies, and so on) prepared at different initial molar ratio of Ce6 to corresponding small molecule (Ce6/NSMs = 1:1, 1:3, 1:5, $1: 8$ ), respectively. Where free NSMs self-assemblies were prepared via same method without the addition of Ce6.
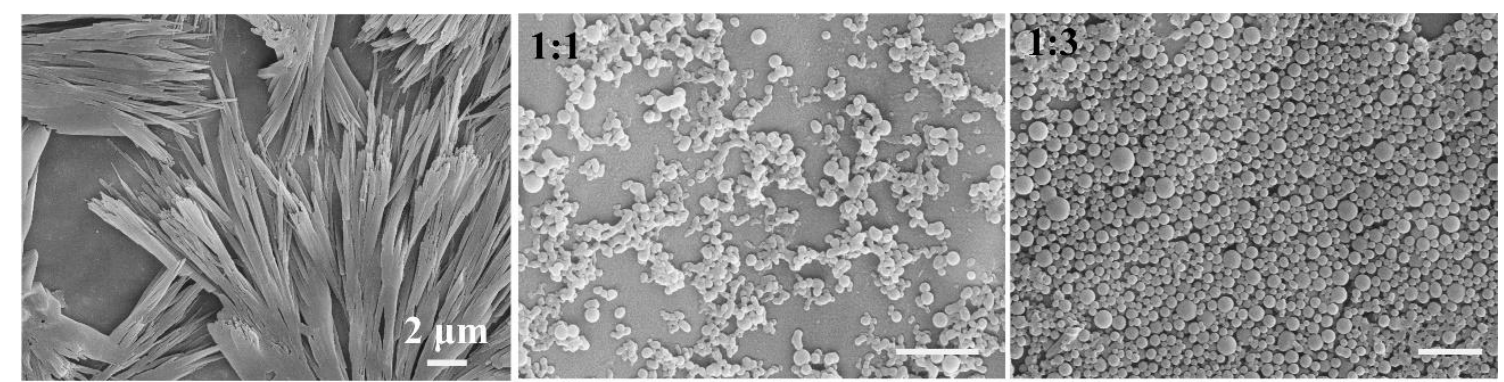

Betulonic acid (BC) Self-assembly

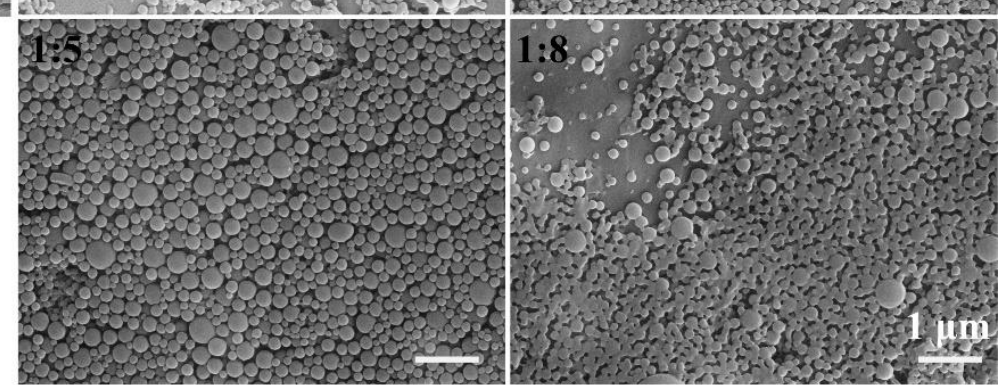

BC-Ce6 co-assemblies

(NPs: 1:1, 1:3, 1:5, 1:8) 
Betulinic acid (BA)

Self-assembly
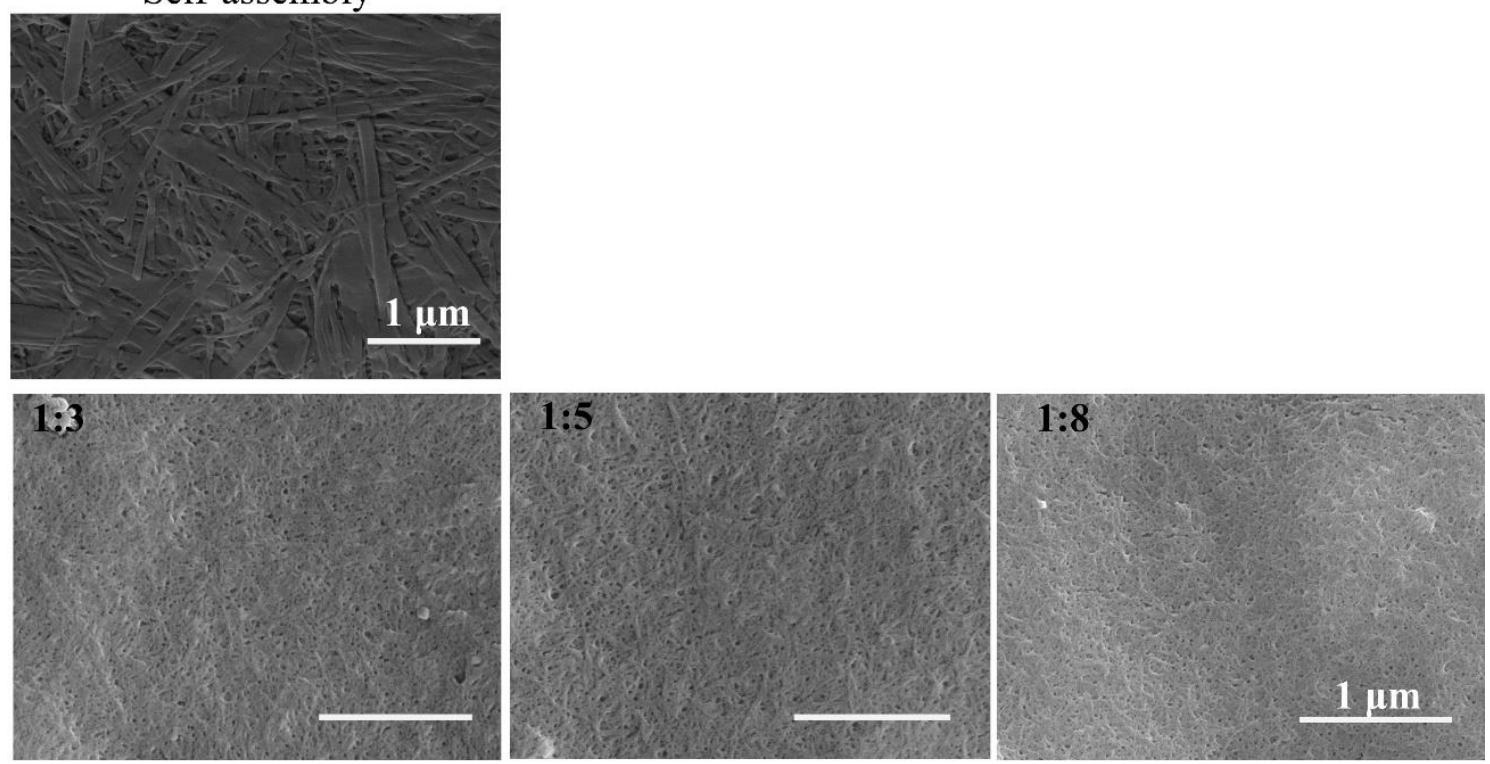

BA-Ce6 co-assemblies

(NPs: 1:3, 1:5, 1:8)

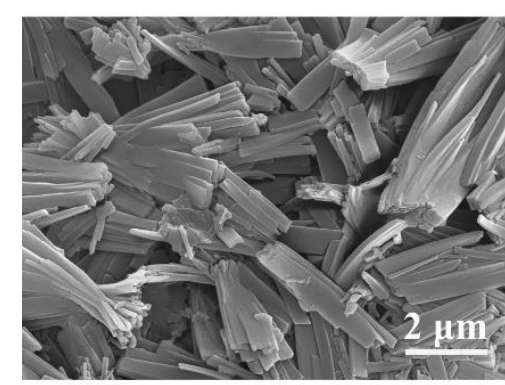

Glycyrrhetinic acid (GA) Self-assembly
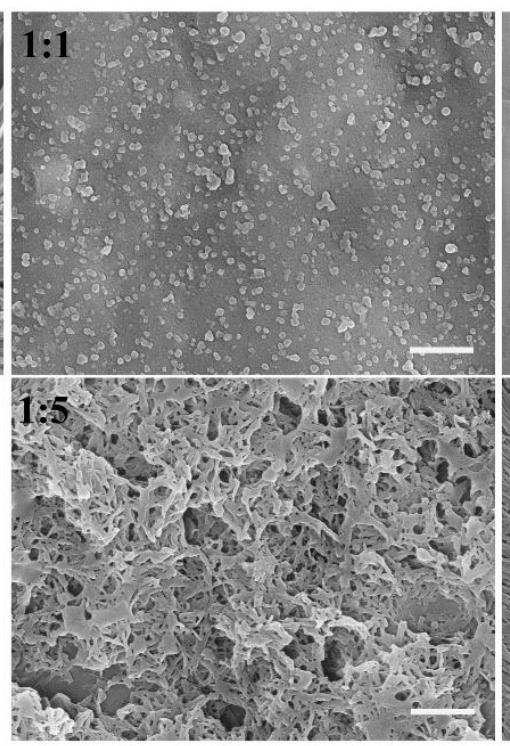

GA-Ce6 co-assemblies

NPs: 1:1, 1:8 


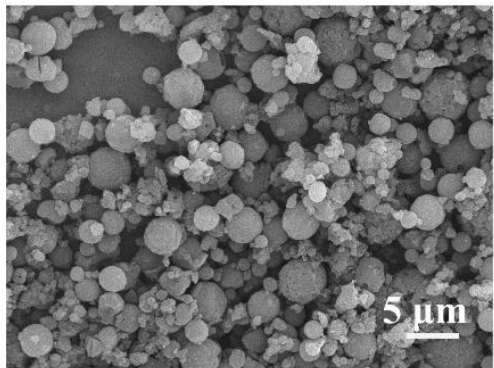

Ursolic acid (UA) Self-assembly
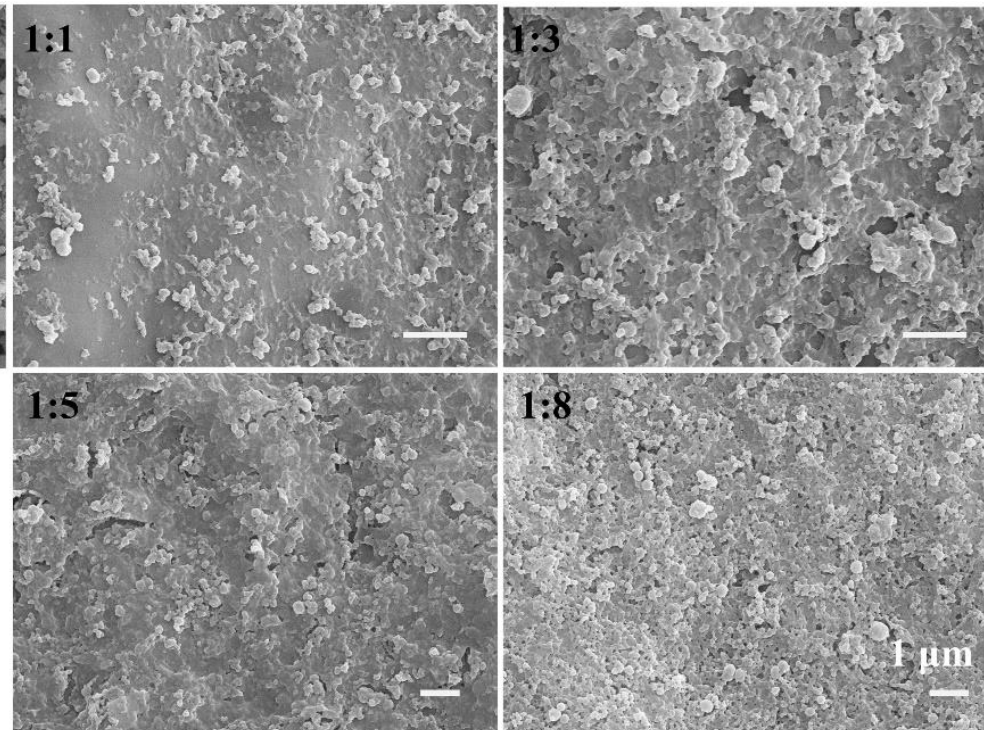

UA-Ce6 co-assemblies

NPs: 1:1, 1:3, 1:5, 1:8

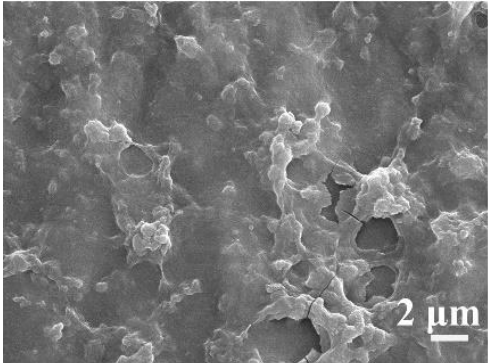

Deoxycholic acid (DCA) assembly

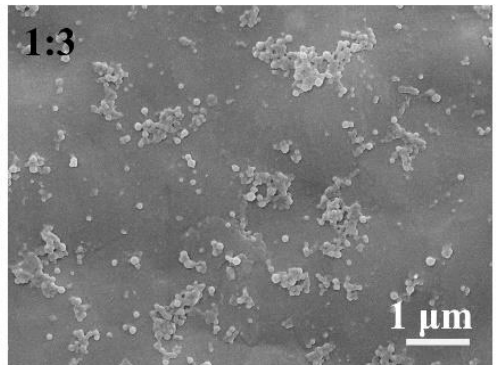

DCA-Ce6 co-assemblies NPs: 1:3

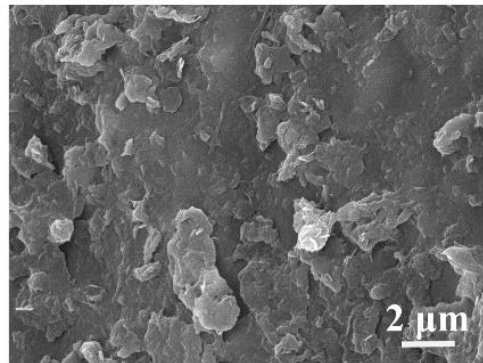

Ursocheoxycholic acid (UDCA) assembly

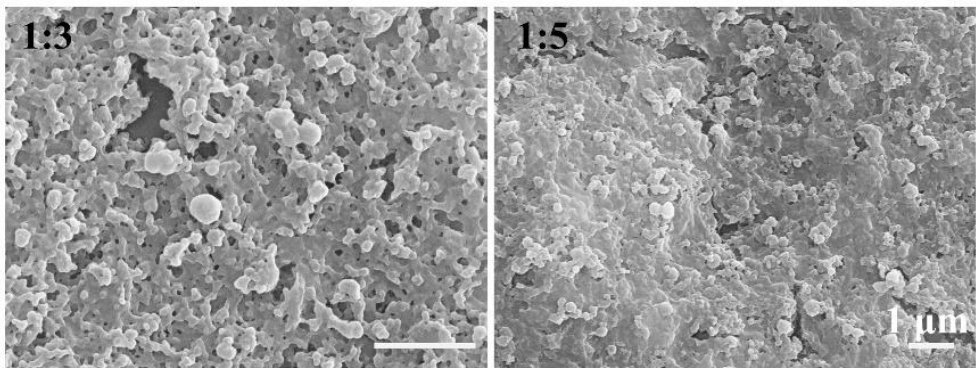

UDCA-Ce6 co-assemblies

NPs: 1:3 
Poricoic acid (PAA)

Self-assembly
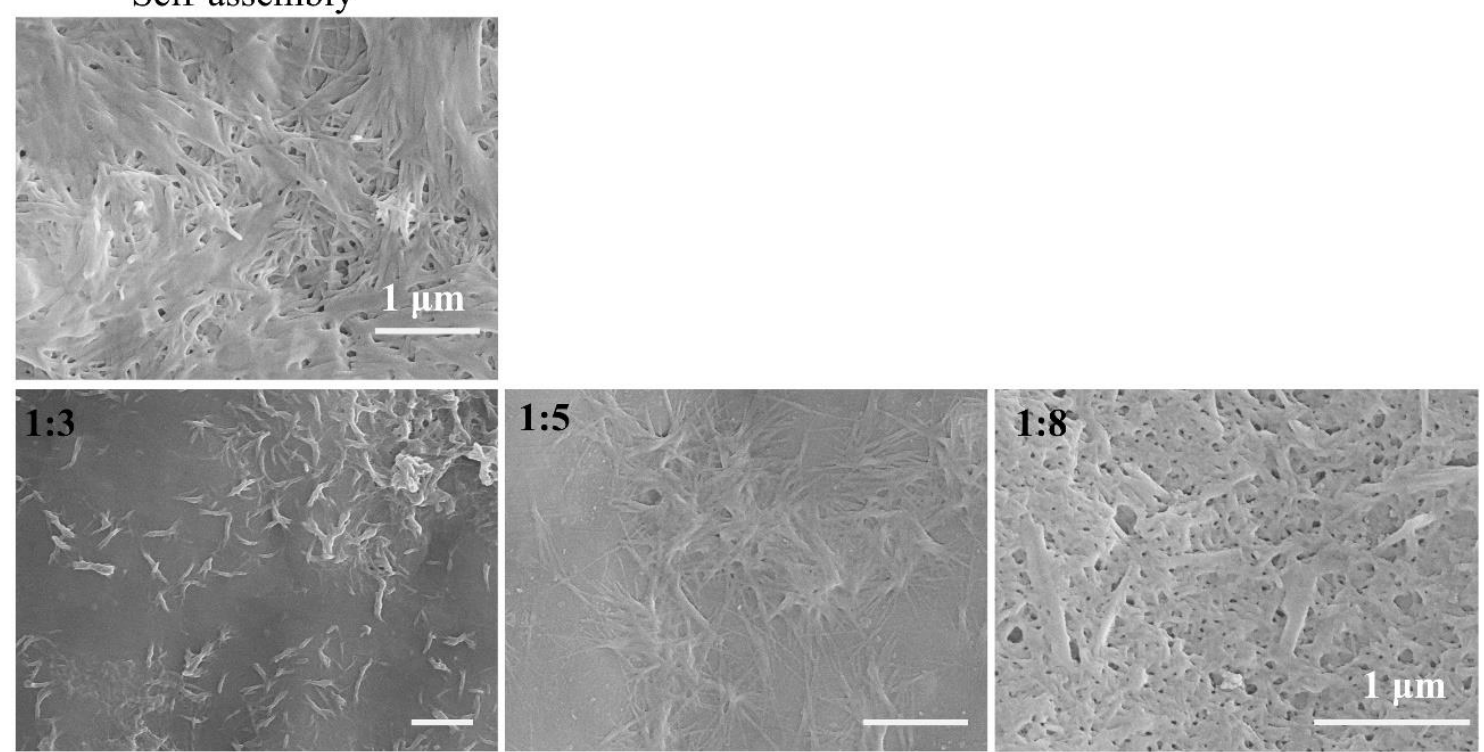

PAA-Ce6 co-assemblies

NPs: 1:3

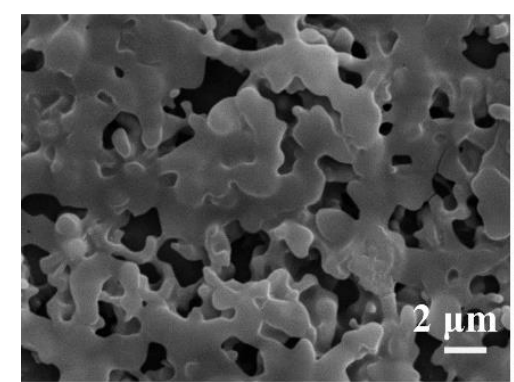

Abietic acid (AA)

Self-assembly

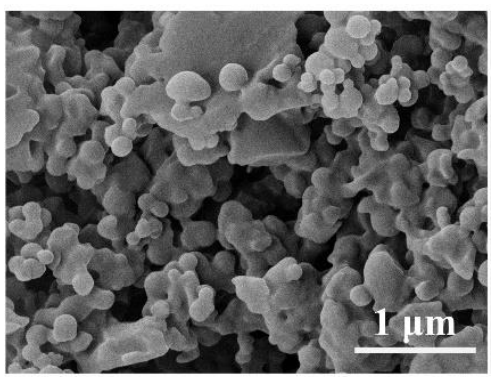

12-Hydroxyabietic acid (12HAA) Self assembly

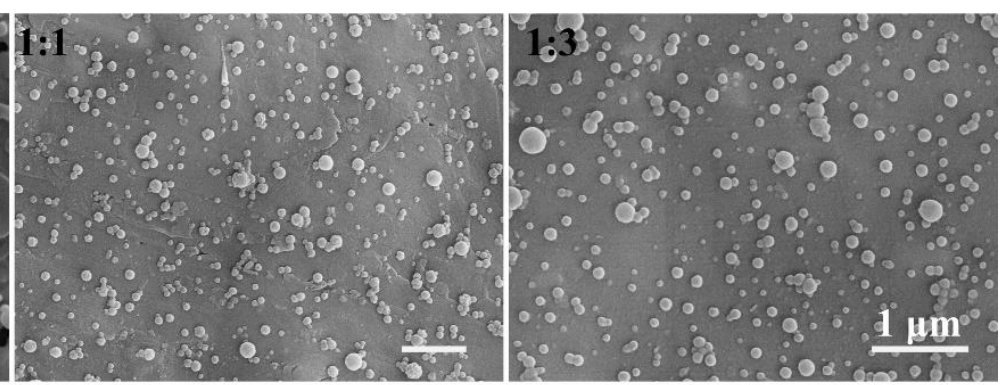

AA-Ce6 co-assemblies

NPs: 1:1, 1:3

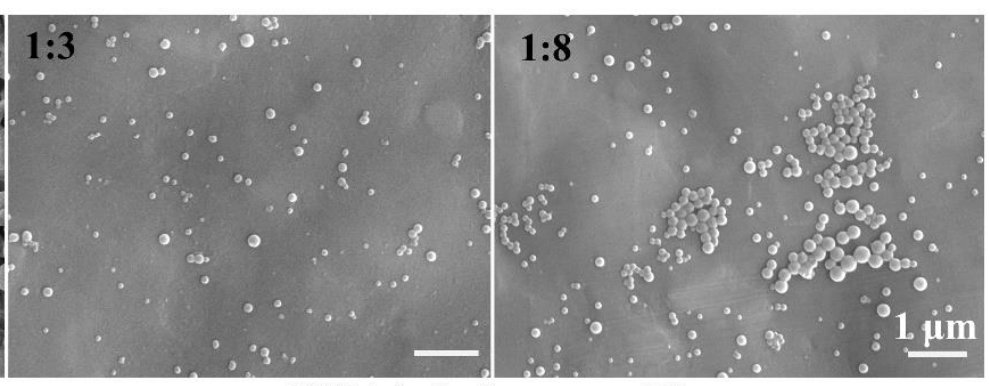

12HAA-Ce6 co-assemblies

NPs: 1:3, 1:8 


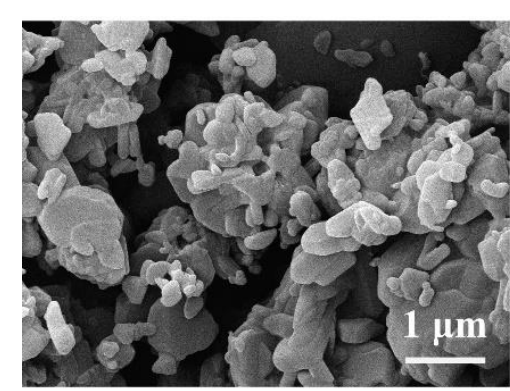

Dehydroabietic acid (DA) Self-assembly

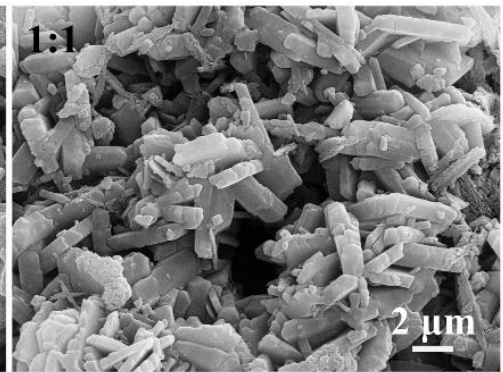

1:5

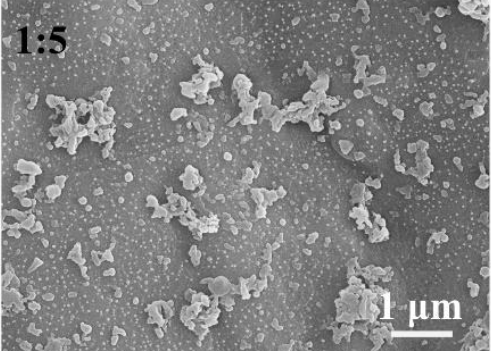

DA-Ce6 co-assemblies

NPs: 1:5, 1:8

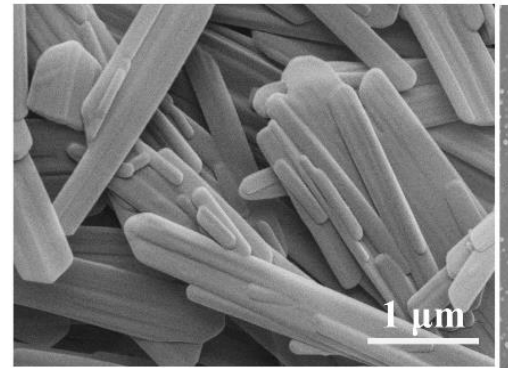

15-Hydroxydehydroabietic acid (15HDA)

Self-assembly
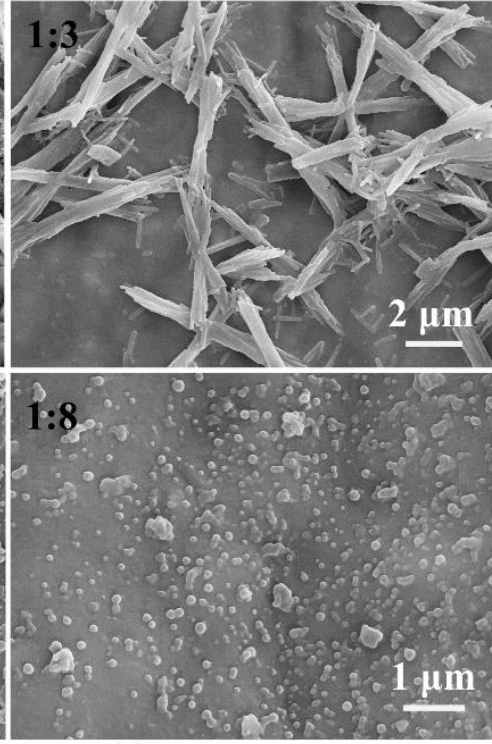

$\mu \mathrm{m}$

$$
\text { s }
$$

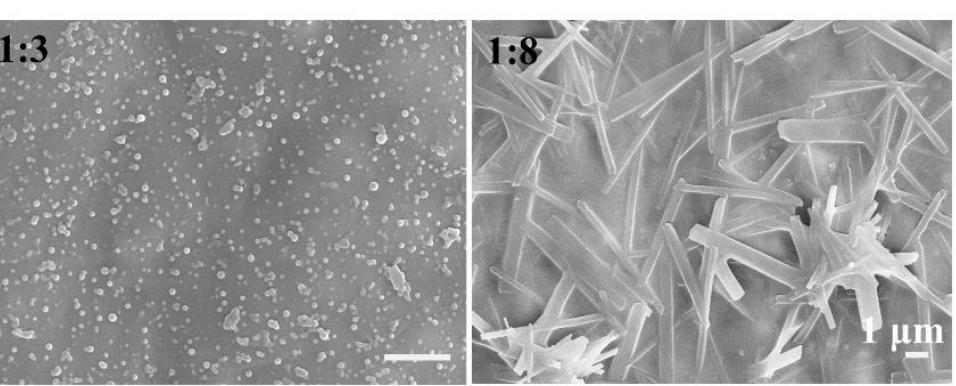

15HDA-Ce6 co-assemblies NPs: 1:3 


\section{Additional Figures}

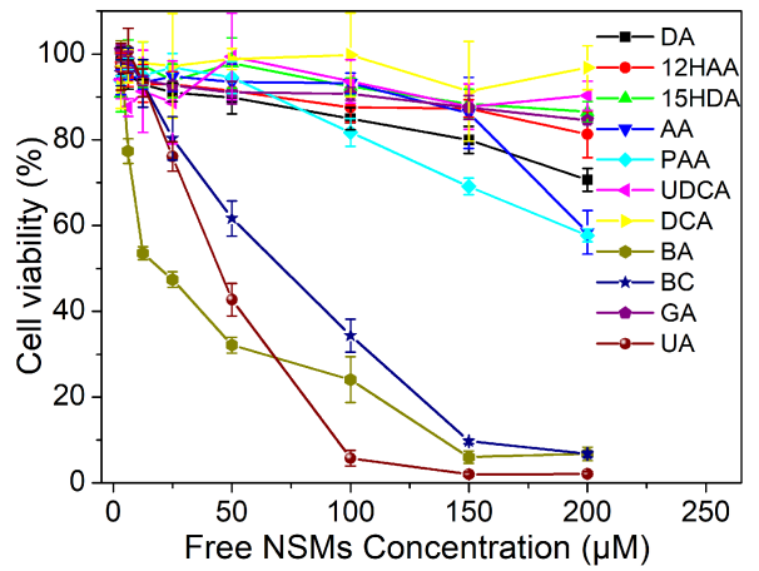

Figure S3. Cell viability of 4T1 cells after incubation with identified eleven free natural small molecules for $24 \mathrm{~h}$, respectively, determined by MTT assay.

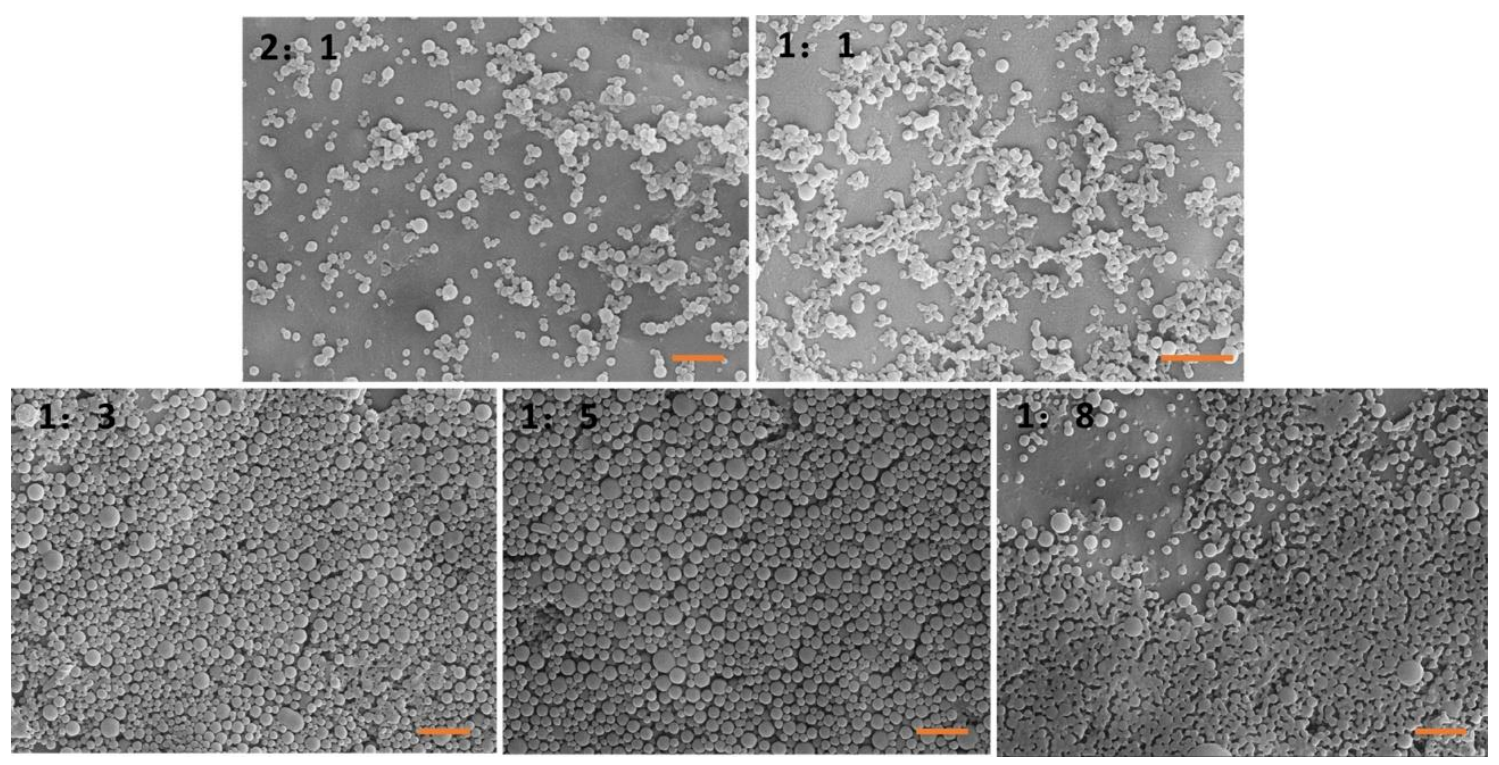

Figure S4. SEM images of separated precipitate BC-Ce6 NPs prepared at different initial molar ratios of Ce6 to BC including 2:1, 1:1, 1:3, 1:5, 1:8, respectively. Scale bar: $1 \mu \mathrm{m}$. 

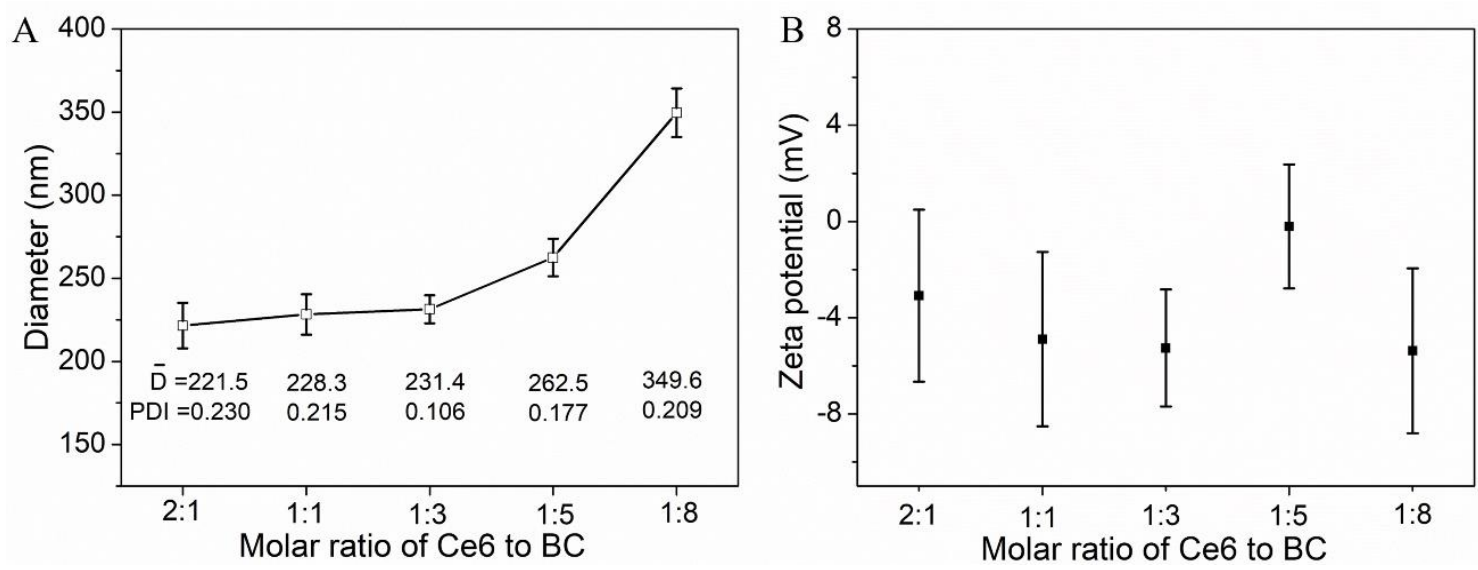

Figure S5. (A) Average DLS diameter, polydispersity value (PDI), and (B) zeta potential of BC-Ce6 NPs prepared at different initial molar ratios of Ce6 to BC.

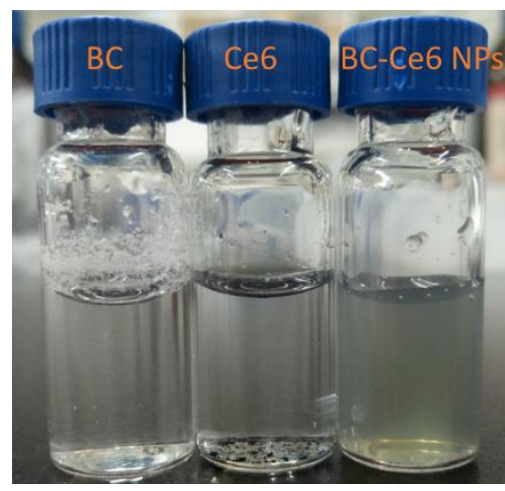

Figure S6. The photographs of free BC, free Ce6 and BC-Ce6 NPs (equivalent Ce6 concentration: $17.5 \mu \mathrm{g} / \mathrm{mL}$ ) in water, respectively. 


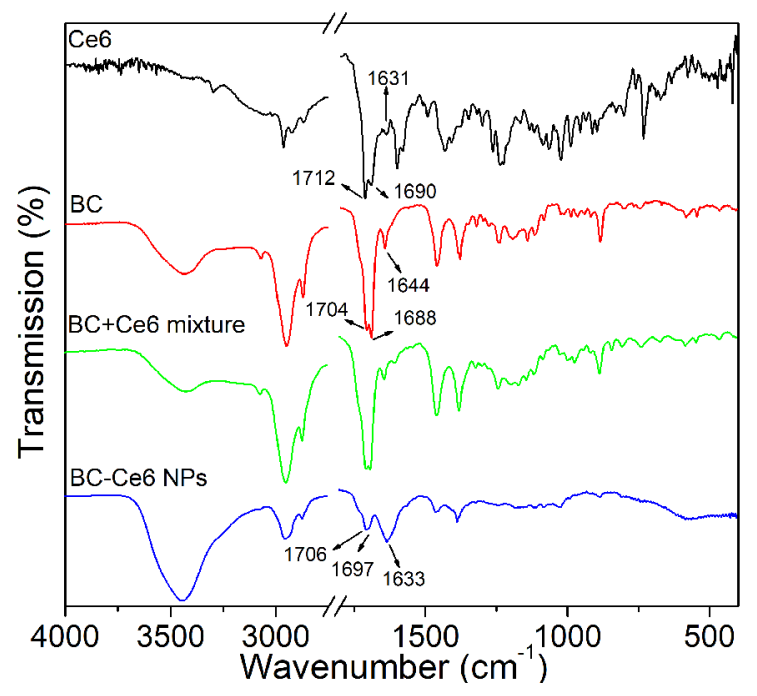

Figure S7. FT-IR spectra of Ce6, betulonic acid (BC), BC+Ce6 mixture, and co-assembled BCCe6 NPs, respectively.

For free Ce6, the absorption peaks at $1712 \mathrm{~cm}^{-1}, 1689 \mathrm{~cm}^{-1}$ are attributed to the stretching vibration of $\mathrm{C}=\mathrm{O}$, and $1631 \mathrm{~cm}^{-1}$ correspond to the characteristic vibration of $\mathrm{C}=\mathrm{N}$. After the formation of co-assembled BC-Ce6 NPs, the characteristic peak of $\mathrm{C}=\mathrm{N}$ appears at $1633 \mathrm{~cm}^{-1}$, demonstrating the successful assembly of BC and Ce6. Meanwhile, different from free BC and their mixture, the stretching vibration of $\mathrm{C}=\mathrm{O}$ in BC-Ce6 NPs shows a slight movement, appearing at $1706 \mathrm{~cm}^{-1}$ and $1697 \mathrm{~cm}^{-1}$, further implying the occurrence of intermolecular interactions in co-assembled NPs.

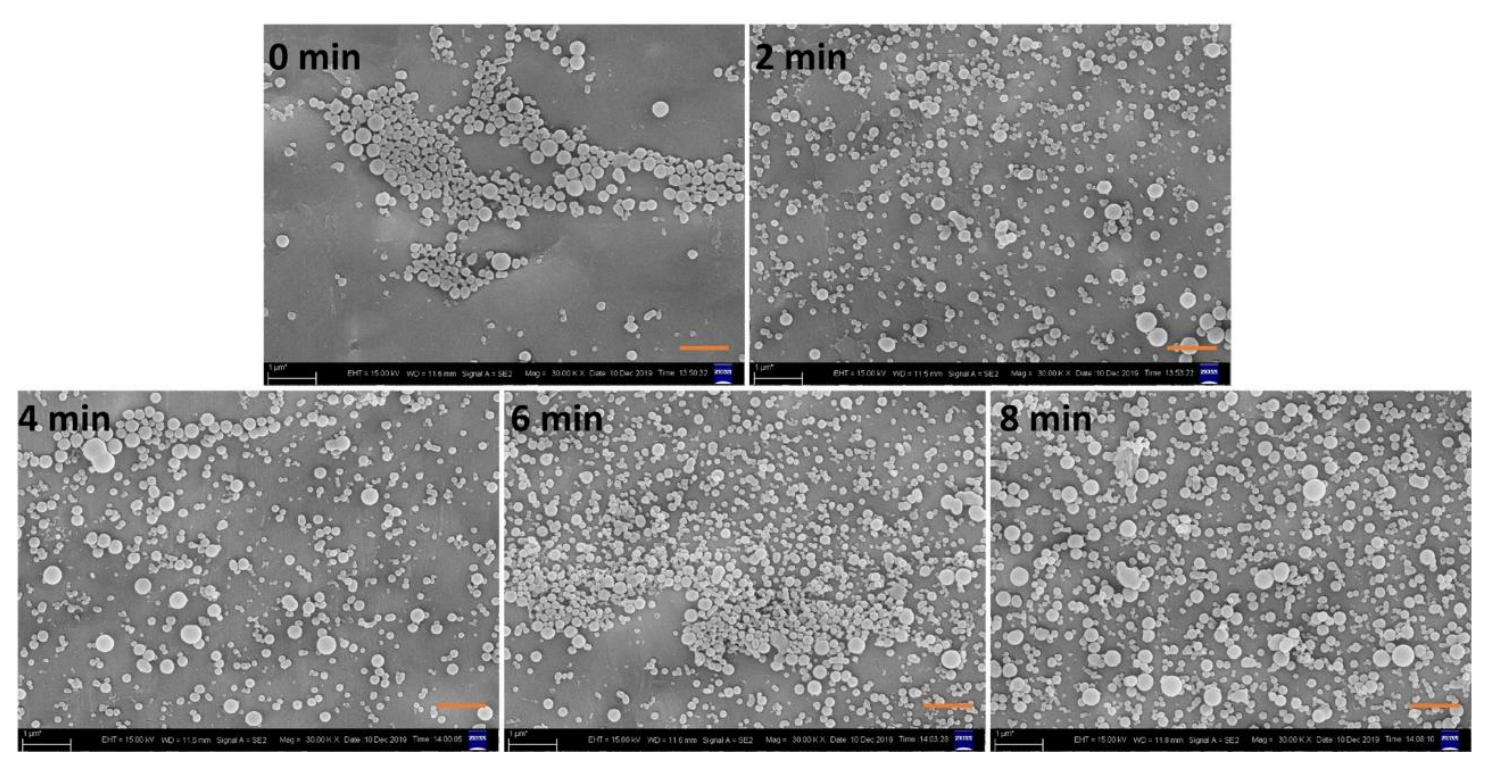

Figure S8. SEM images of the separated precipitates BC-Ce6 NPs after continuous sonication of the reaction mixture of $\mathrm{Ce} 6$ and $\mathrm{BC}$ in water for various times $(0,2,4,6,8 \mathrm{~min})$ and followed by centrifugation, using Ce6 and BC (molar ratio 1:3) as building blocks. Scale bar: $1 \mu \mathrm{m}$. 

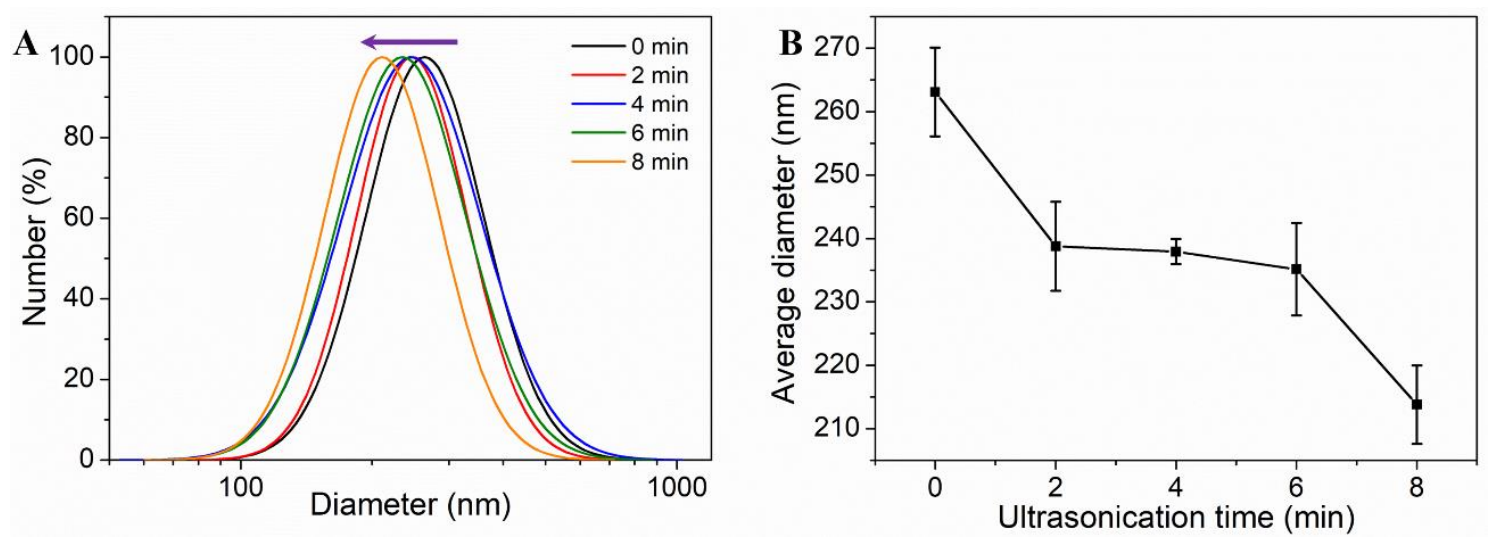

Figure S9. (A) DLS profiles and (B) corresponding average diameter of BC-Ce6 NPs using $\mathrm{Ce} 6$ and $\mathrm{BC}$ (molar ratio 1:3) as building blocks, and prepared by ultrasonication the reaction mixture of $\mathrm{Ce} 6$ with $\mathrm{BC}$ for various times $(0,2,4,6,8 \mathrm{~min})$ by the following centrifugation.
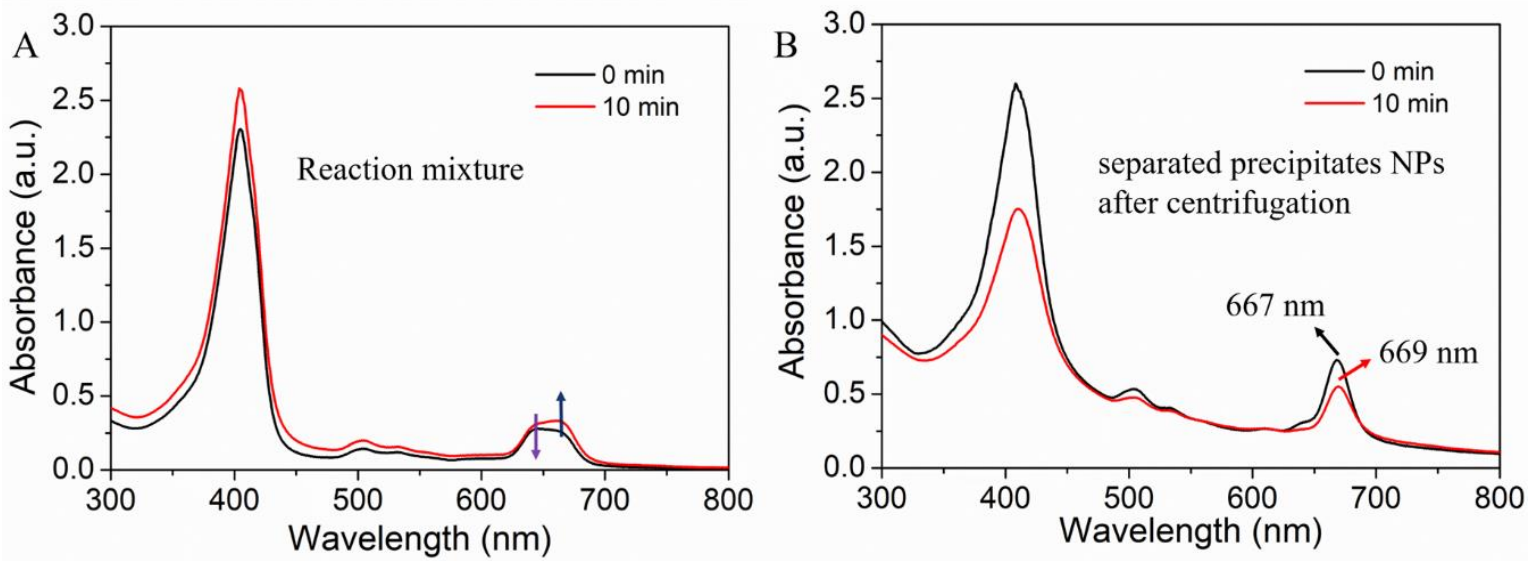

Figure S10. Uv-vis absorption spectra of the (A) directed reaction mixture and (B) separated BC-Ce6 NPs by centrifugation before and after sonication for $10 \mathrm{~min}$, respectively. NPs also prepared using $\mathrm{Ce} 6$ and $\mathrm{BC}$ (molar ratio 1:3) as building blocks.

As shown in Figure S10A, in the UV absorption of reaction solution, the relative absorbance of the broad peak around $665 \mathrm{~nm}$ increases with the introduction of sonication, while the peak around $640 \mathrm{~nm}$ shows a falling trend. Meanwhile, the corresponding separated precipitates NPs exhibit a slight red-shift (2 nm) from $667 \mathrm{~nm}$ to $669 \mathrm{~nm}$ (Figure S10B). These results indicated that continuous ultrasound promoted and enhanced intermolecular interactions between $\mathrm{Ce} 6$ and BC. Furthermore, even if the reaction mixture is not treated with ultrasound $(0 \mathrm{~min})$, the obtained NPs still show a red-shifted Qy band $(667 \mathrm{~nm})$ than free Ce6 $(665 \mathrm{~nm})$, suggesting a strong intermolecular interaction between these two species to promote the co-assembly easily. 


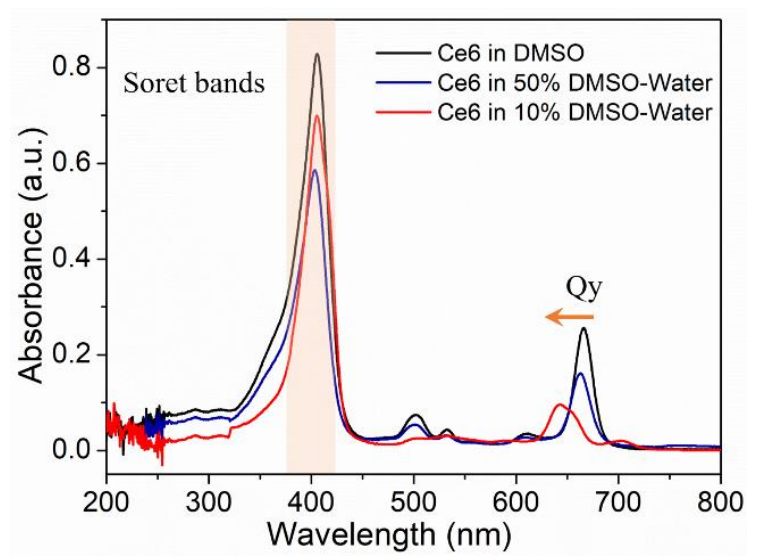

Figure S11. Uv-vis absorption spectra of monomeric Ce6 in DMSO, Ce6 aggregates in 50\% DMSO-Water, and 10\% DMSO-Water at equivalent Ce6 concentration of $4.0 \mu \mathrm{g} / \mathrm{mL}$, respectively.

Ce6 prefers to exist as monomer state in soluble DMSO media, while it presents at H-type aggregates (dimer or multimer) in a form of co-facial arrangement in insoluble or high polarity media (e.g., aqueous solution). ${ }^{13,14}$ As the increase of water proportion, the Qy bands exhibited significant blue-shift with reduced absorbance, while no obvious shift of Soret bands in Ce6 aggregates could be observed, suggesting the formation of H-type aggregates. 

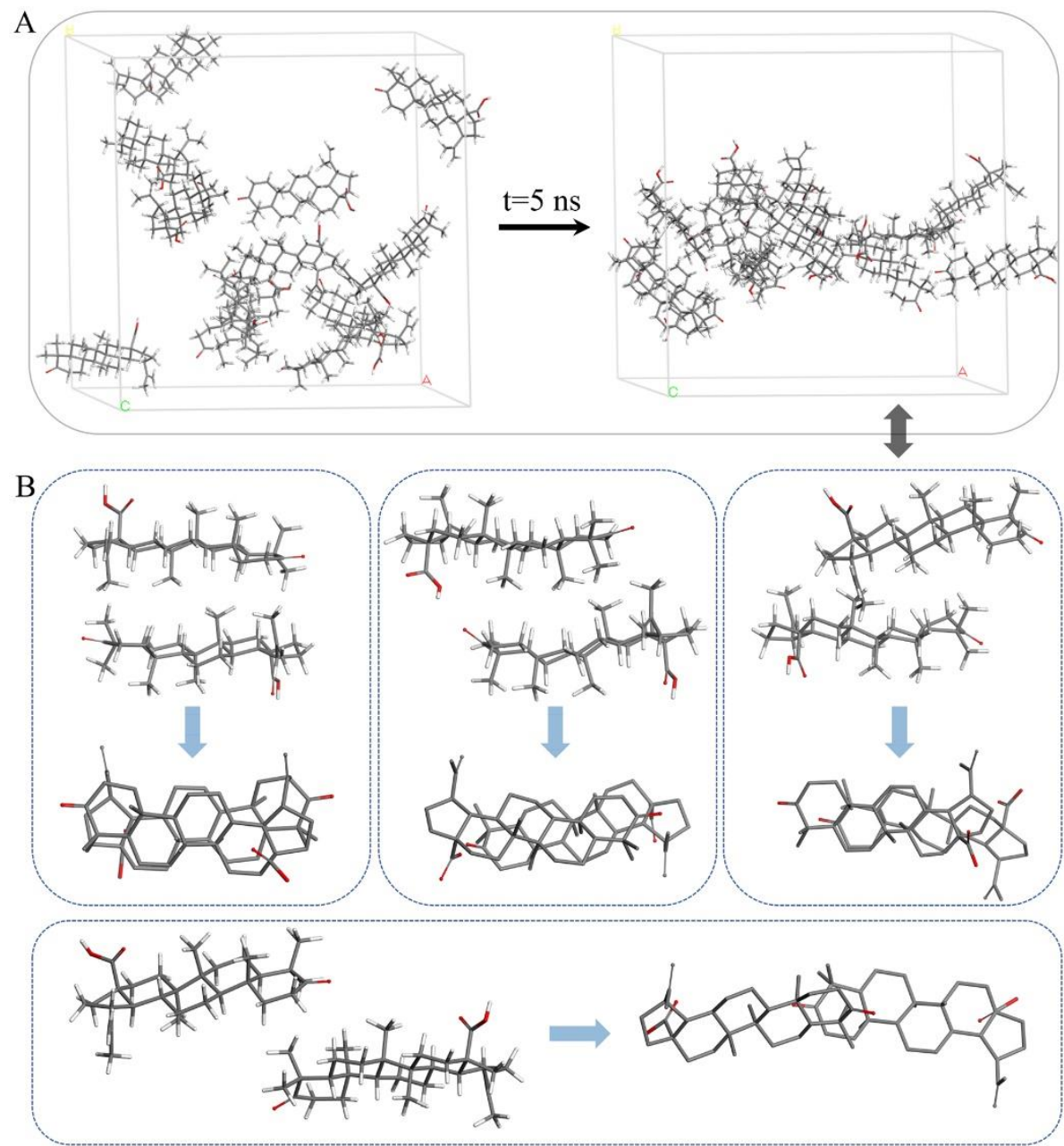

Figure S12. (A) Structure of free BC self-assemblies obtained by MD simulation for a total of $5 \mathrm{~ns}$ (5000 ps). Water molecules are omitted. (B) The obtained BC dimeric are amplified in the boxes. BC prefers to exist as dimeric in a form of linear coplanar arrangement with a crossed hydrophobic ring, and these linear BC dimeric were further grown to form rod-like BC selfassemblies, showing a certain regular configuration. 


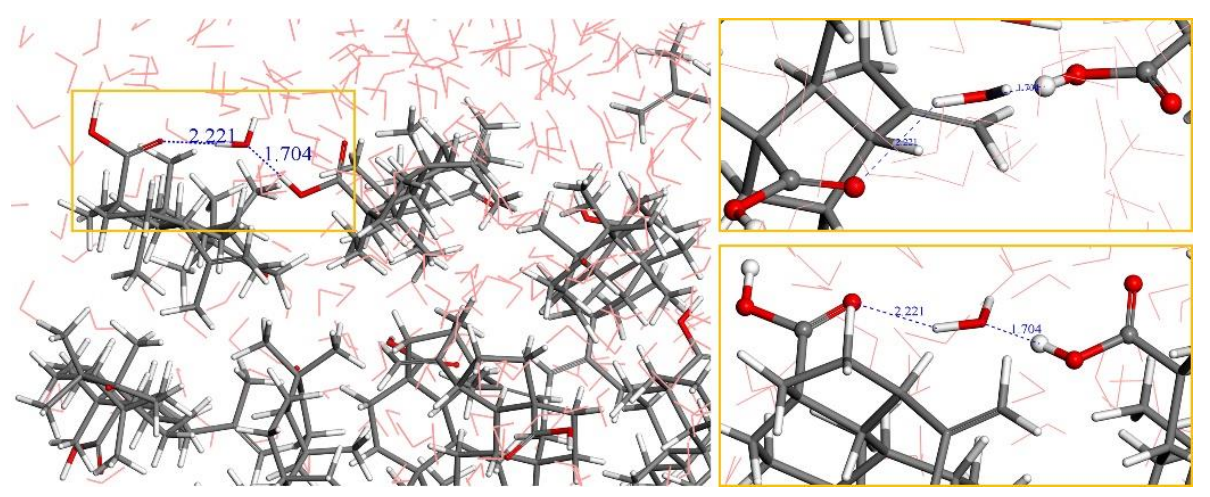

Figure S13. Views of water bridge structure in BC self-assemblies. BC formed a water bridge through one water molecule by two hydrogen bonds. The line and stick models were separately used for the $\mathrm{H}_{2} \mathrm{O}$ and $\mathrm{BC}$ molecules.

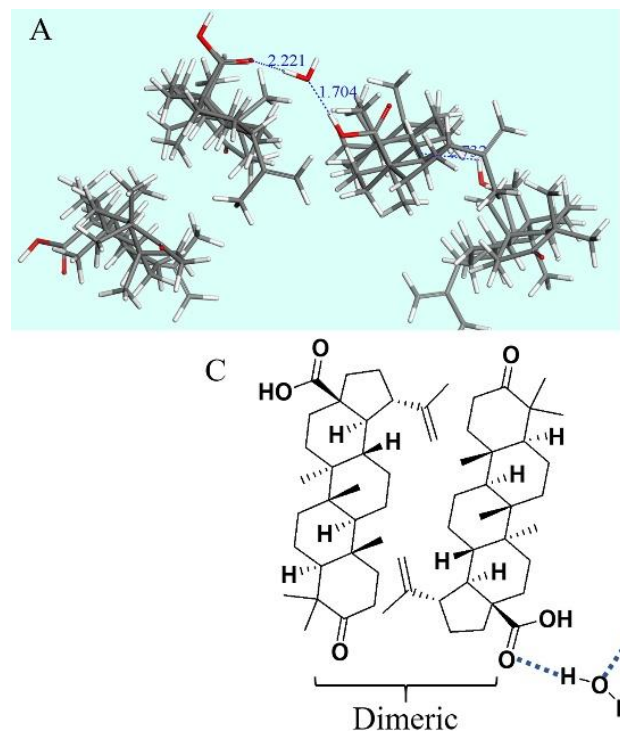

B
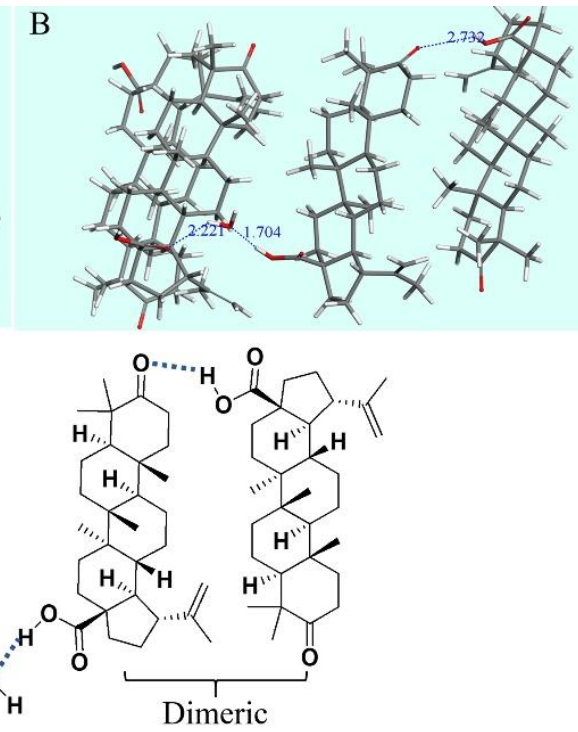

Figure S14. (A) Front view, (B) top view, and (C) corresponding molecule structure of two pairs of BC dimeric. A possible intermolecular hydrogen bond exhibited in BC dimeric, and a water bridged structure by two hydrogen bonds exist in two pair of dimeric. 


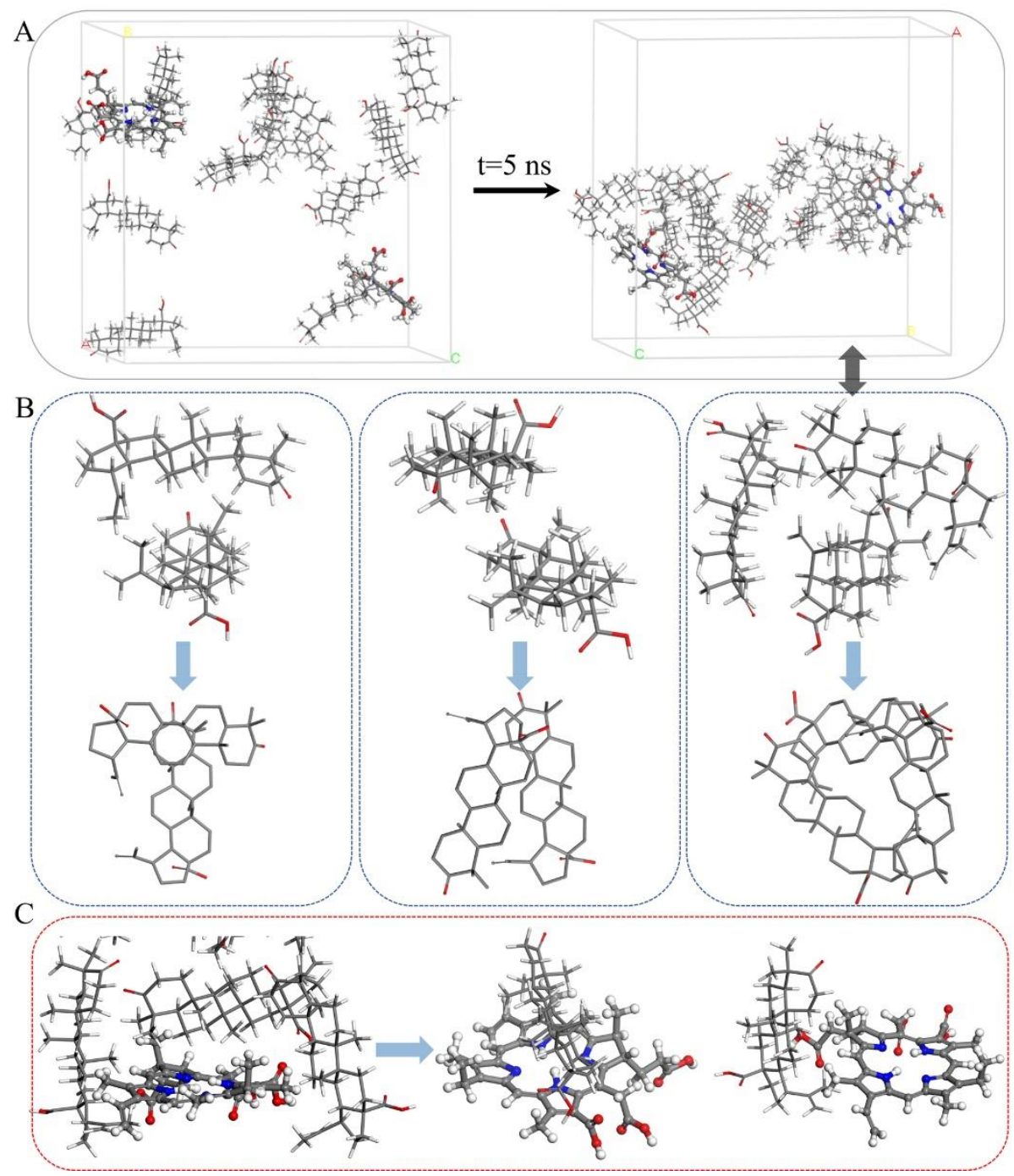

Figure S15. (A) Structure of co-assembled BC-Ce6 NPs obtained by MD simulation for a total of $5 \mathrm{~ns}(5000 \mathrm{ps})$. Water molecules are omitted. (B) The possible BC dimeric in a form of coplanar arrangement are amplified in the boxes, showing a disordered arrangement. (C) The possible interactions model between $\mathrm{Ce} 6$ and $\mathrm{BC}$ are amplified in the boxes. The line and ball models were separately used for the $\mathrm{BC}$ and $\mathrm{Ce} 6$ molecules. 

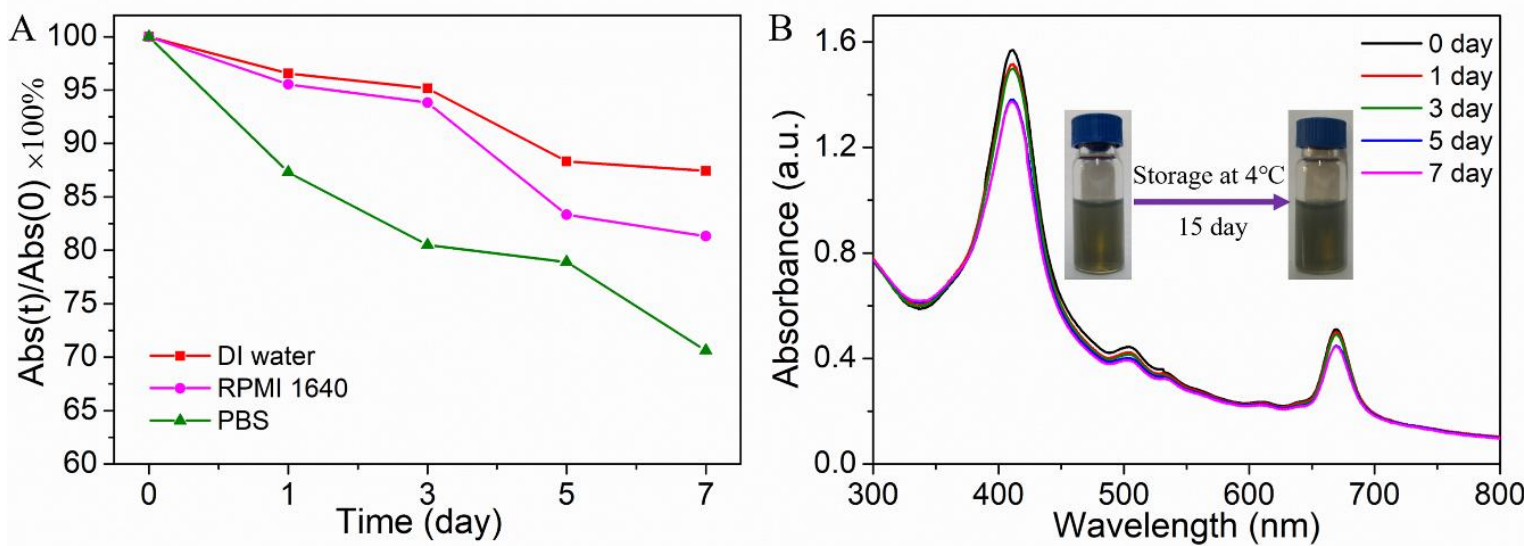

Figure S16. Stability of BC-Ce6 NPs. (A) Degradation process over time (t) of BC-Ce6 NPs incubated in DI water, RPMI 1640 and PBS, as measured by their optical absorption at $409 \mathrm{~nm}$, and expressed as $\operatorname{Abs}(\mathrm{t}) / \operatorname{Abs}(0) \times 100 \%$, where $\operatorname{Abs}(\mathrm{t})$ and $\operatorname{Abs}(0)$ is represent the absorbance of NPs at day t and day 0, respectively. (B) Uv-vis absorption spectra of BC-Ce6 NPs over time in DI water, and insets display the state of BC-Ce6 NPs storage at $4^{\circ} \mathrm{C}$ for 15 days.

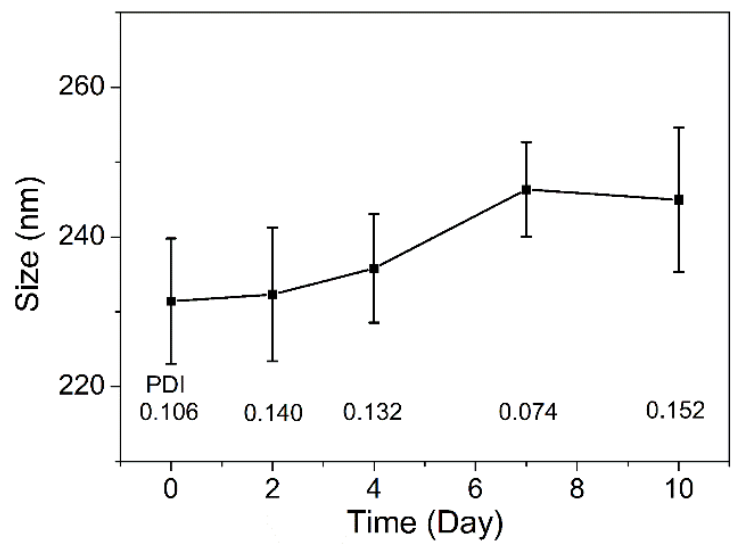

Figure S17. Size change of BC-Ce6 NPs over time in PBS (pH 7.4) solution. No significant size changes were found, indicating the better stability of BC-Ce6 NPs. The slight increase in average size may be due to nano-aggregation induced by small size effect.
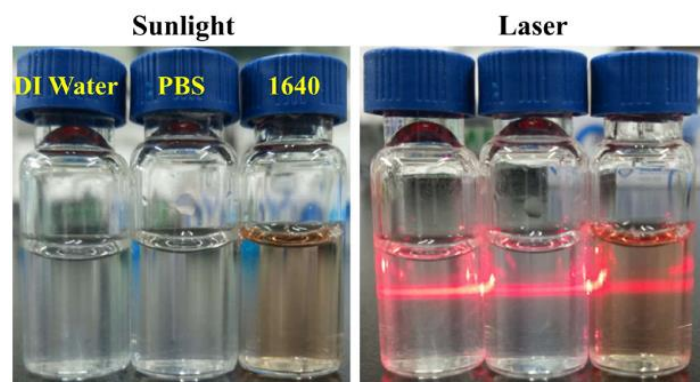

Figure S18. The photographs in the sunlight of BC-Ce6 NPs dispersed in different media including water, PBS, and 1640 culture medium. After the dispersion passed through with red laser light, an obvious Tyndall effect could be observed, indicating the colloidal nature of BCCe6 NPs. 


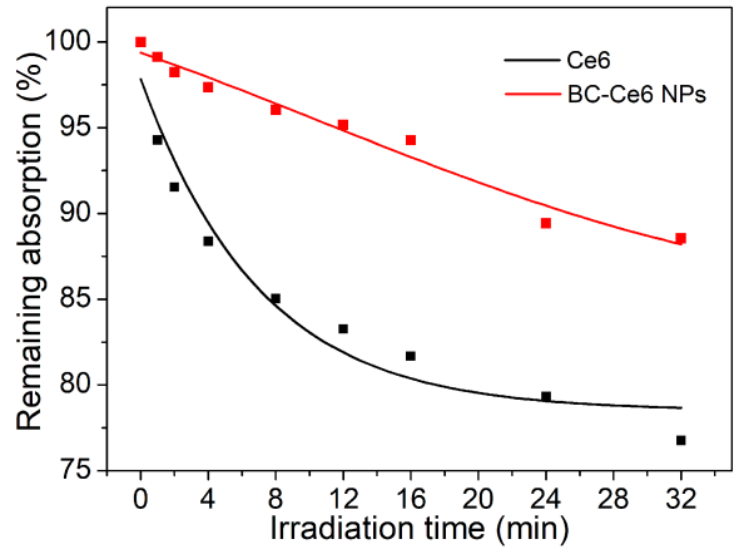

Figure S19. The photobleaching plots of free Ce6 and BC-Ce6 NPs under continuous light irradiation within $32 \mathrm{~min}$. The remaining absorption (\%) at their Qy bands were utilized to evaluate the anti-photobleaching ability. The remaining absorption ratio of BC-Ce6 NPs and Ce6 were separately $88.5 \%$ and $76.8 \%$ even after prolonged irradiation for 32 min, implying the enhanced anti-photobleaching ability and good photostability of BC-Ce6 NPs.

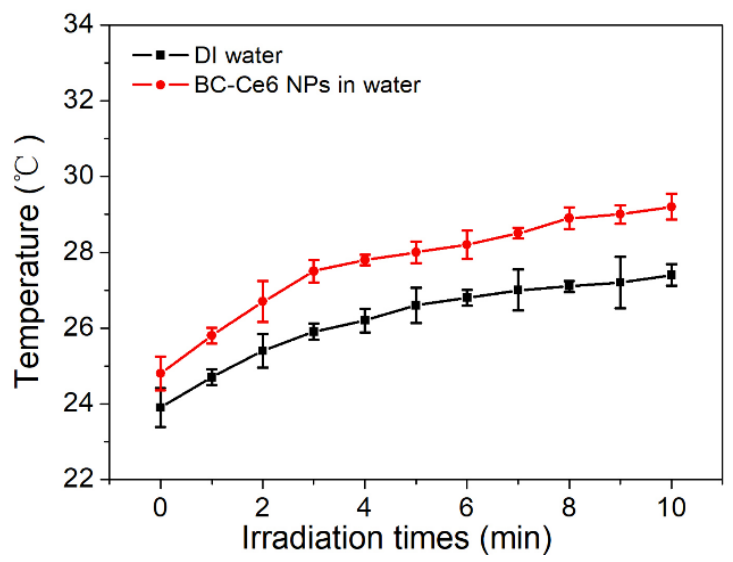

Figure S20. Temperature change of BC-Ce6 NPs $(56 \mu \mathrm{g} / \mathrm{mL})$ under continuous light irradiation $(808 \mathrm{~nm}, 1.5 \mathrm{~W})$ with $10 \mathrm{~min}$, as measured by Infrared Thermometer (TiS40, Fluke, USA). 

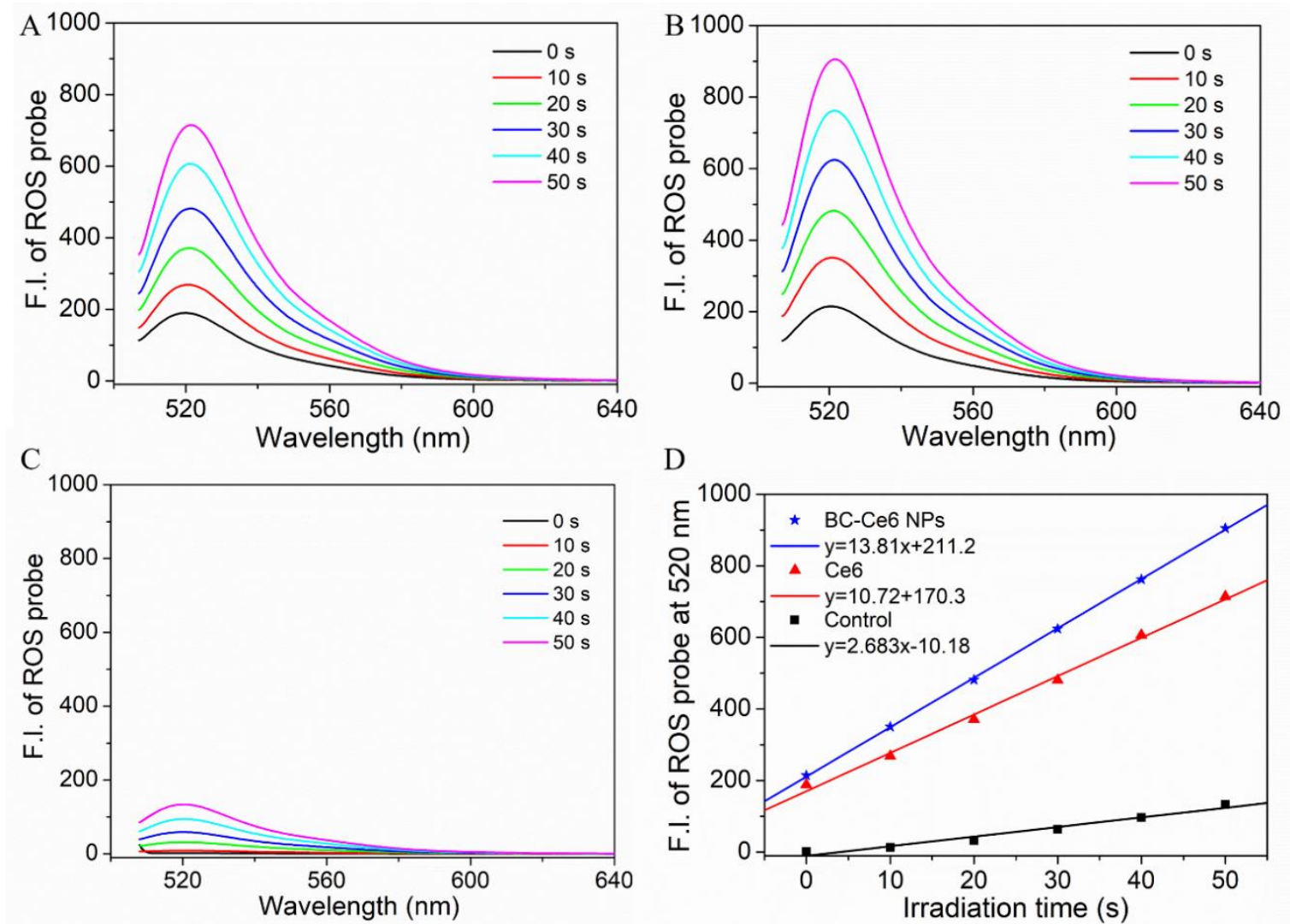

Figure S21. The total ROS generation of (A) Ce6, (B) BC-Ce6 NPs, (C) Control group (same concentration of DCFH) in water with final Ce6 equivalent concentration of $2.5 \mu \mathrm{g} / \mathrm{mL}$, was detected by monitoring the fluorescence intensity (F.1) change of 2,7-dichlorofluorescin $(30 \mu \mathrm{M}$, DCFH probe) at $520 \mathrm{~nm}$ after irradiation for various times. (D) The F.l. comparison of ROS probe at different light points. For fluorescence, the excitation wavelength is $488 \mathrm{~nm}$. 

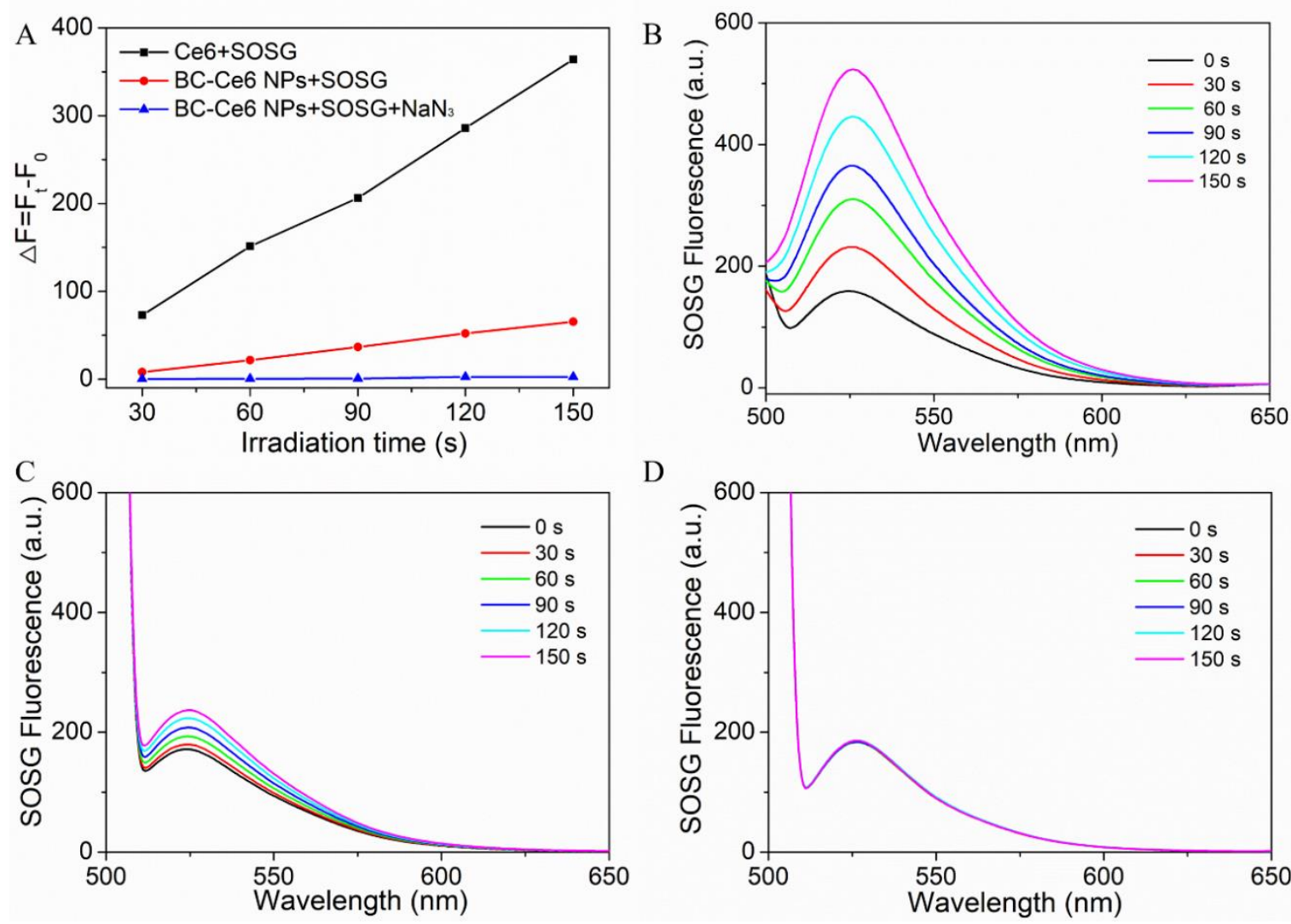

Figure S22. The ${ }^{1} \mathrm{O}_{2}$ generation of BC-Ce6 NPs was evaluated by using a singlet oxygen fluorescence probe SOSG. (A) Comparison of ${ }^{1} \mathrm{O}_{2}$ probe fluorescence intensity changes $\left(\Delta \mathrm{F}=\mathrm{F}_{\mathrm{t}}-\mathrm{F}_{0}\right)$ at $527 \mathrm{~nm}$ after irradiation of $\mathrm{Ce} 6$ in water, BC-Ce6 NPs in water, and BC-Ce6 $\mathrm{NPs}+\mathrm{NaN}_{3}$ for various times. And corresponding fluorescence spectra of SOSG induced by (B) Ce6, (C) BC-Ce6 NPs, (D) BC-Ce6 NPs+NaN 3 , respectively. Where $\mathrm{F}_{\mathrm{t}}$ and $\mathrm{F}_{0}$ separately represent the fluorescence intensity of SOSG at time $t$ and time $0 . \mathrm{NaN}_{3}$ was used as a specific quencher to ${ }^{1} \mathrm{O}_{2}$. The final equivalent $\mathrm{Ce} 6$ concentration is $2 \mu \mathrm{g} / \mathrm{mL}$. For fluorescence, the excitation wavelength is $488 \mathrm{~nm}$.
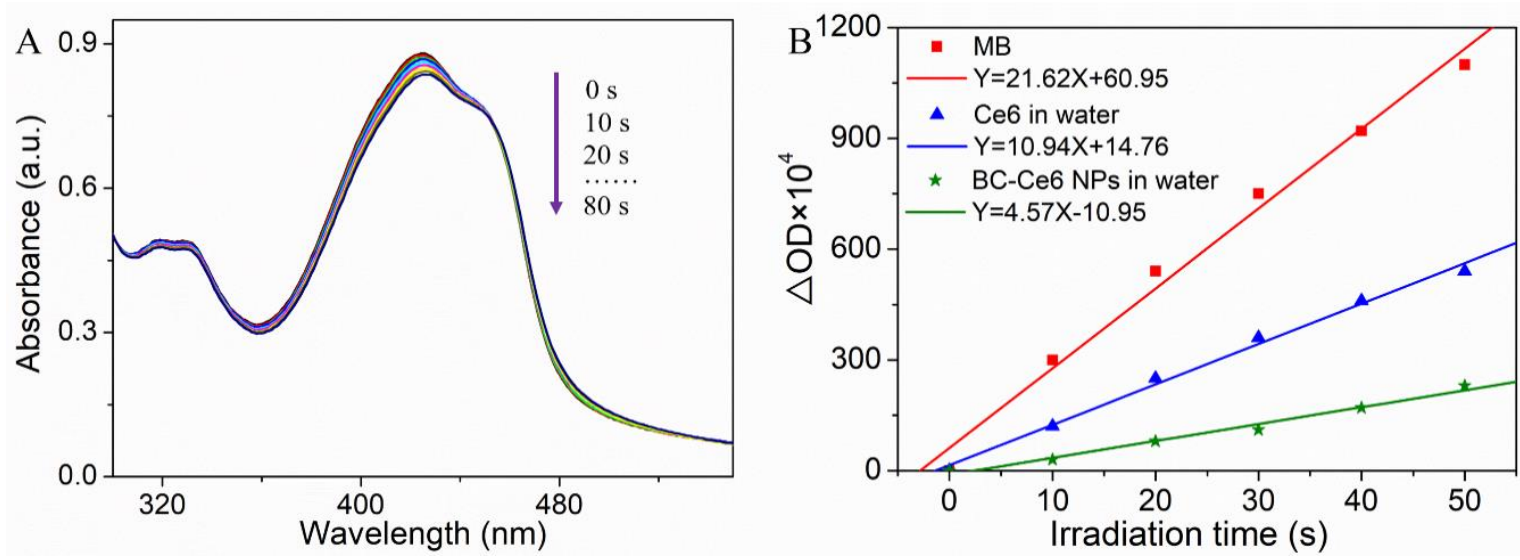

Figure S23. (A) Uv-vis spectra of photodecomposition of DPBF induced by ${ }^{1} \mathrm{O}_{2}$ generating from irradiation of BC-Ce6 NPs, and (B) First-order plots the photodecomposition of DPBF changes $(\triangle \mathrm{OD})$ at $422 \mathrm{~nm}$ under irradiation of MB in DMSO, Ce6 in water, BC-Ce6 NPs in water within $50 \mathrm{~s}$, respectively. Where methylene blue $(\Phi=0.52)$ in DMSO was used as the reference compound. 

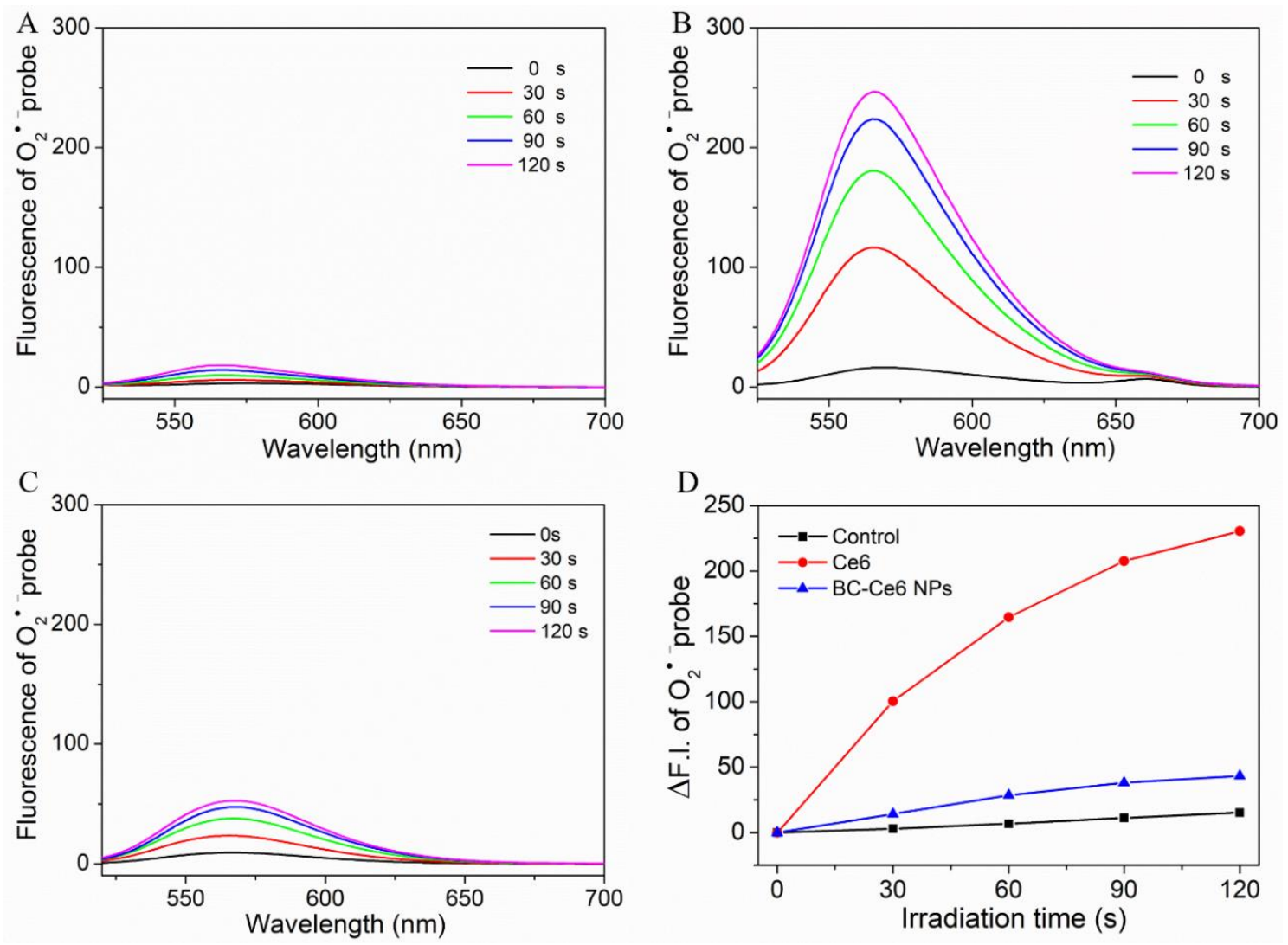

Figure S24. The superoxide anion $\left(\mathrm{O}_{2}{ }^{-}{ }^{-}\right)$generation of BC-Ce6 NPs was evaluated by using a hydroethidine $(\mathrm{HE}, 25 \mu \mathrm{M})$ as a probe combined with ctDNA $(200 \mu \mathrm{g} / \mathrm{mL})$. The fluorescence spectra of $\mathrm{HE}$ after irradiation of (A) Control group only with HE and ctDNA, (B) Ce6 and (C) BC-Ce6 NPs (equivalent Ce6: $2 \mu \mathrm{g} / \mathrm{mL}$ ) in water for different times, respectively. And corresponding (D) fluorescence intensity changes $(\Delta \mathrm{F} .1$. $)$ of $\mathrm{O}_{2}{ }^{*}$ probe at $566 \mathrm{~nm}$ with irradiation times. For fluorescence, the excitation wavelength is $510 \mathrm{~nm}$. 

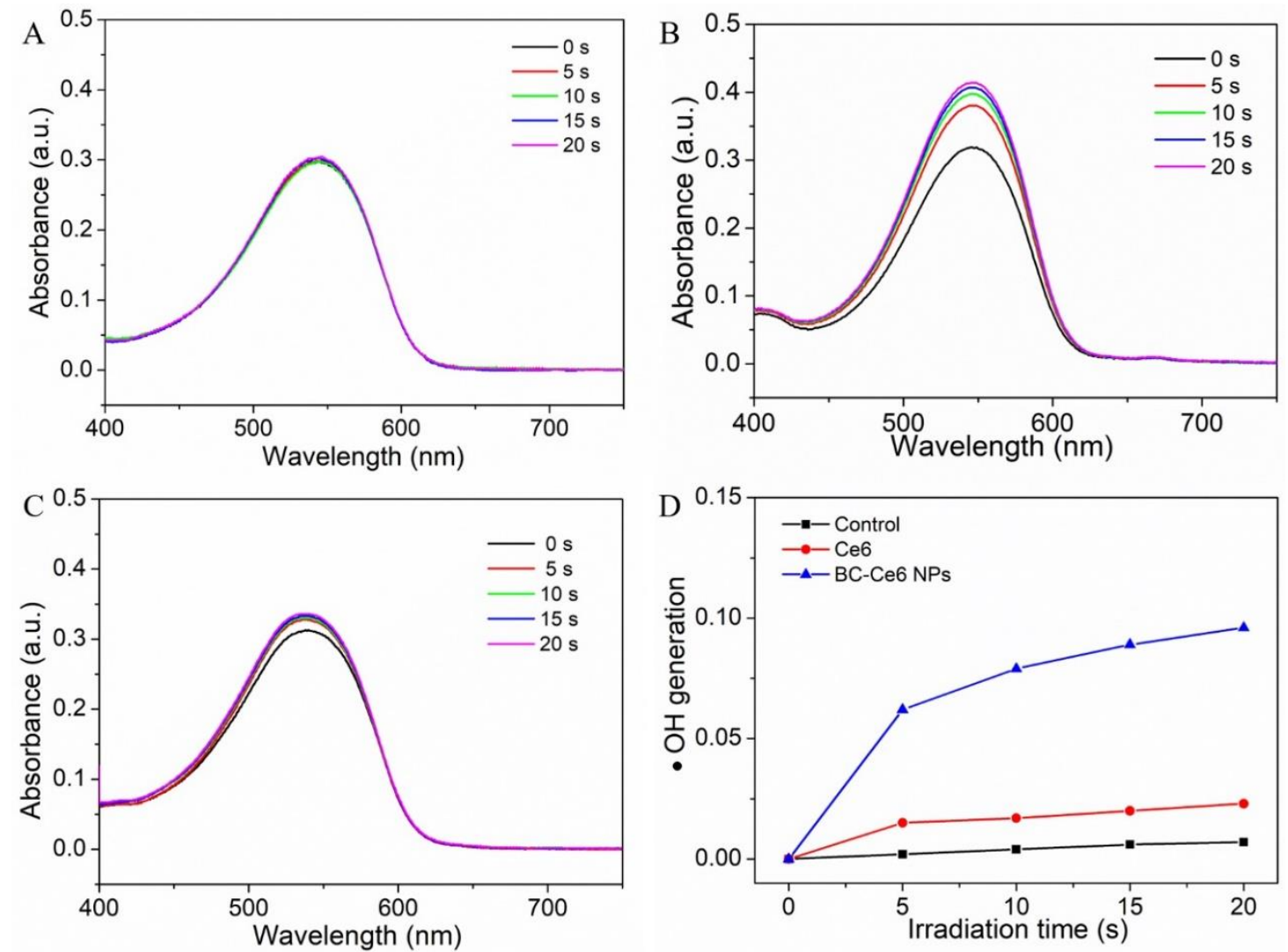

Figure S25. The hydroxyl radicals $(-\mathrm{OH})$ generation was detected based on the chromogenic reaction of Griess reagent using $\cdot \mathrm{OH}$ assay Kit. The Uv-vis absorption spectra of $\cdot \mathrm{OH}$ probe after irradiation of (A) control, (B) BC-Ce6 NPs in water, (C) free Ce6 in water for different times, respectively. And (D) Comparison the absorbance changes around $548 \mathrm{~nm}$ of $\cdot \mathrm{OH}$ probe, where $\operatorname{Abs}(\mathrm{t})$ and $\operatorname{Abs}(0)$ separately represent the absorbance after irradiation for $\mathrm{t} \mathrm{s} 0 \mathrm{~s}$. For detection, the final equivalent $\mathrm{Ce} 6$ concentration is $2 \mu \mathrm{g} / \mathrm{mL}$. 


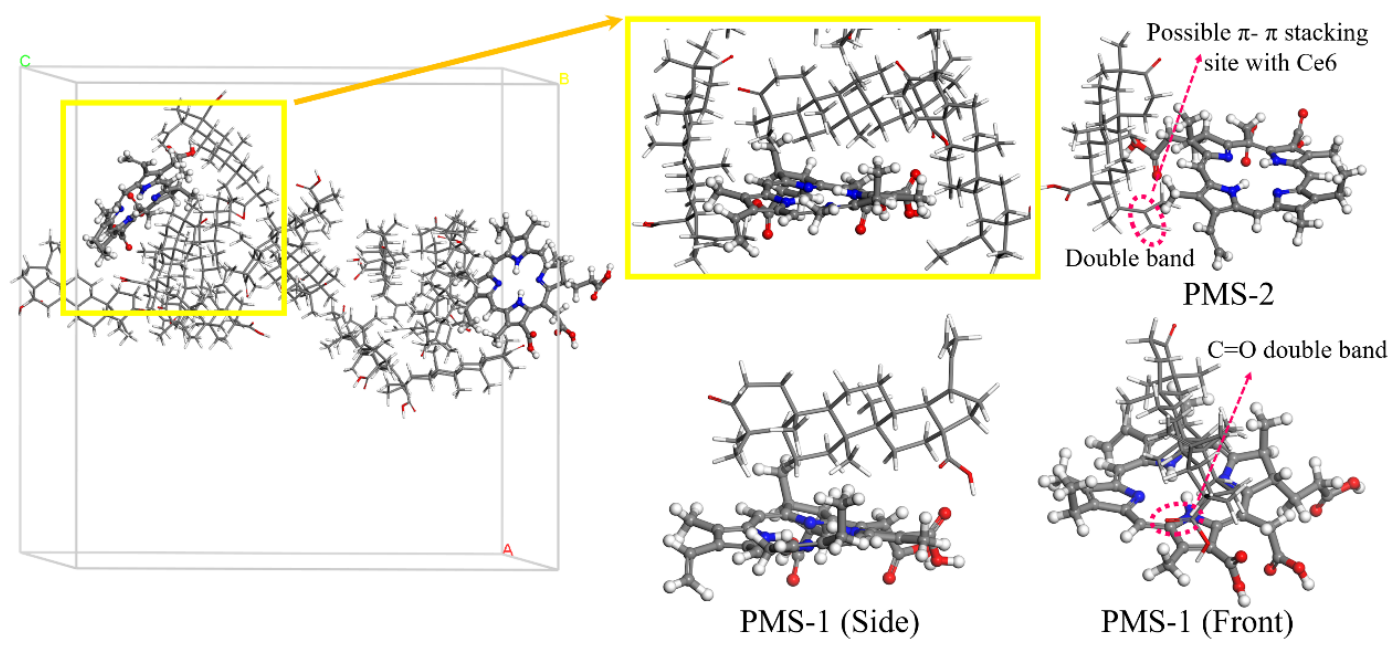

Figure S26. Structure of co-assembled BC-Ce6 NPs by MD simulation. Water molecules are omitted, and the key positions of typical binding sites of possible interactions model between Ce6 and BC (PMS-1, PMS-2) are amplified in the boxes. The line and ball models were separately used for the $\mathrm{BC}$ and $\mathrm{Ce} 6$ molecules. The Uv absorption wavelength ( $\lambda$ max) of PMS1 and PMS-2 is $580.42 \mathrm{~nm}$ and $579.05 \mathrm{~nm}$, respectively, as calculated by DFT.

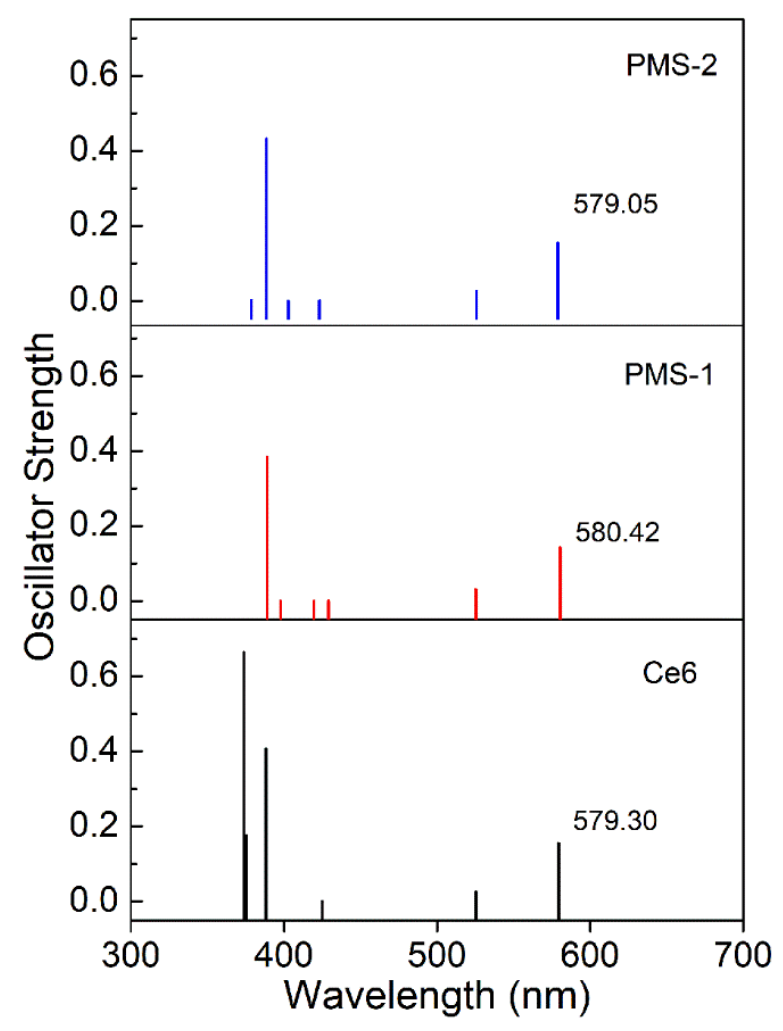

Figure S27. The predicted UV-vis absorption spectra transitions (vertical bars) of free Ce6, PMS-1, PMS-2 calculated by TD-DFT, respectively. 


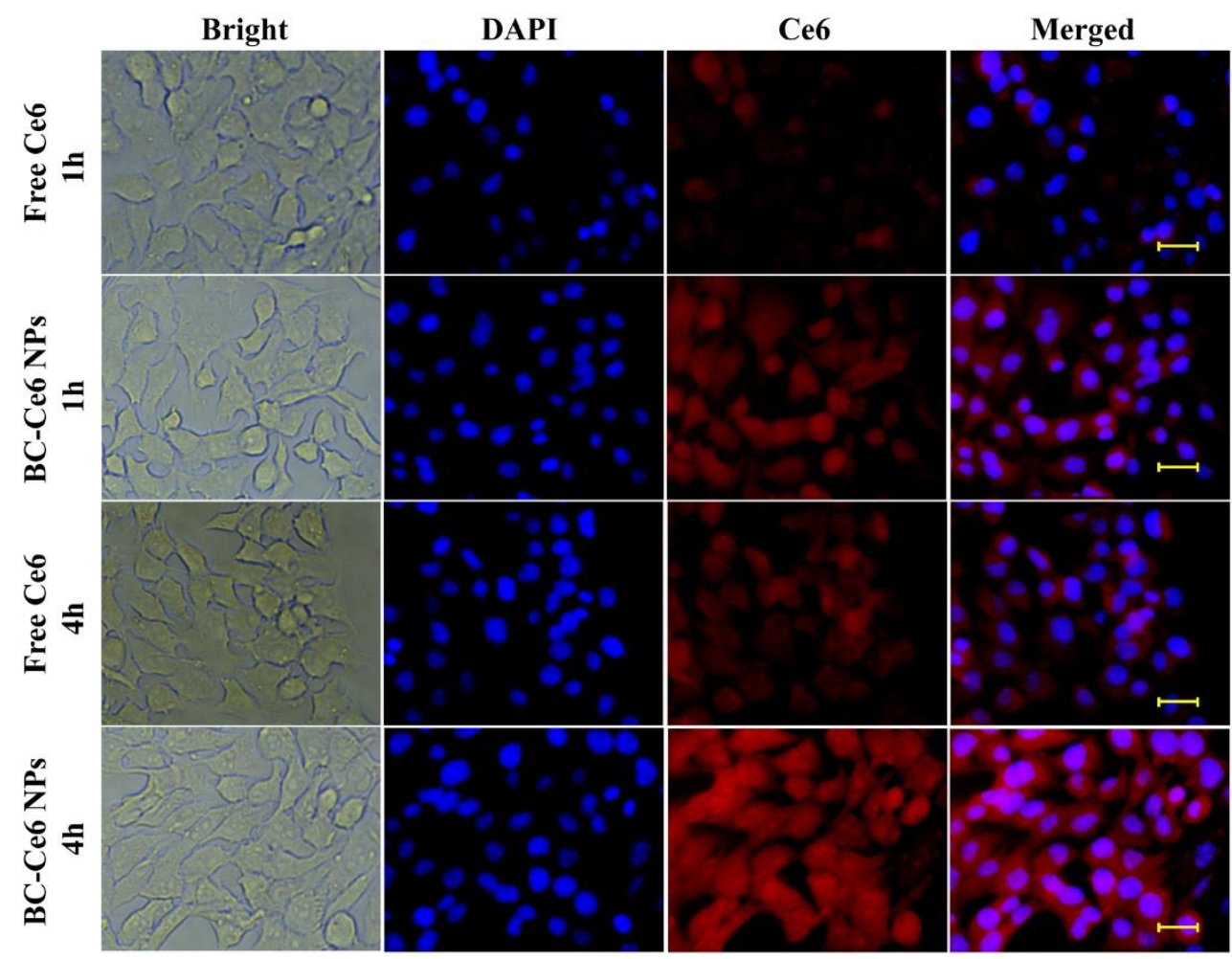

Figure S28. Fluorescence inverted microscopic images of 4T1 cells incubated with BC-Ce6 NPs (equivalent concentration of Ce6: $2 \mu \mathrm{g} / \mathrm{mL}$ ) and free $\mathrm{Ce} 6$ for $1 \mathrm{~h}$ and $4 \mathrm{~h}$. The nucleus was stained with DAPI showing blue fluorescence and excited by Uv excitation; Ce6 was excited by green light showing red fluorescence, which may be the disassemble of BC-Ce6 NPs and released into the cytoplasm, showing a cytoplasmic staining. Scale bar: $20 \mu \mathrm{m}$.

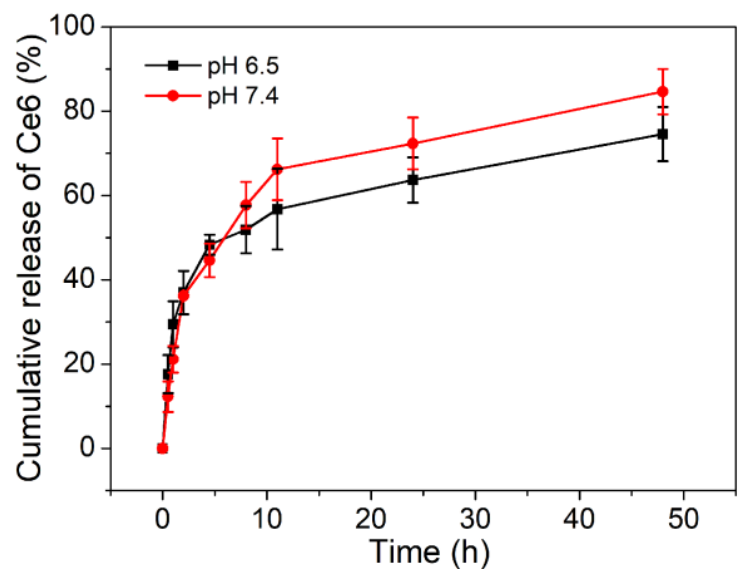

Figure S29. In vitro Ce6 release profiles from BC-Ce6 NPs in PBS (pH 6.5 and 7.4) containing $0.05 \%(\mathrm{v} / \mathrm{v})$ Tween 80 with error bars representing the standard deviation $(\mathrm{n}=3)$ at $37{ }^{\circ} \mathrm{C}$. 


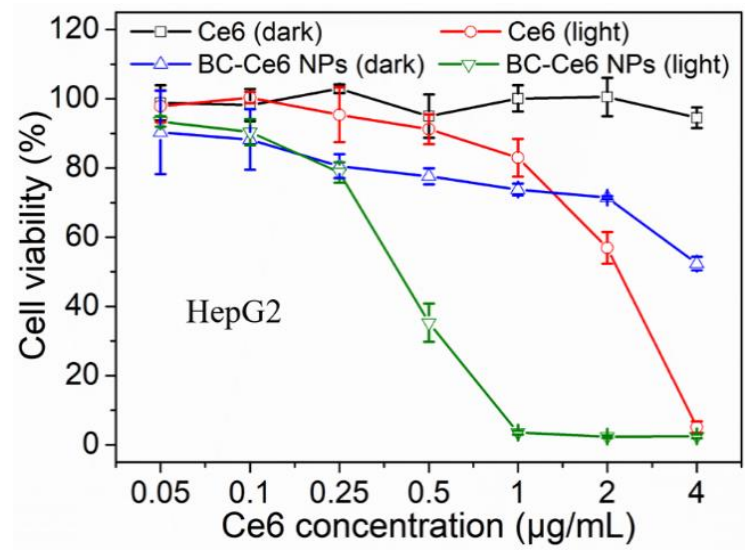

Figure S30. Cell viability of HepG2 cells after incubation with BC-Ce6 NPs and free Ce6 at an equivalent concentration of $\mathrm{Ce} 6(0.05-4 \mu \mathrm{g} / \mathrm{mL})$, which was calculated by the $17.2 \%$ loading of Ce6 in co-assembled NPs. Date represent mean \pm standard deviation $(\mathrm{SD}, \mathrm{n}=6)$.
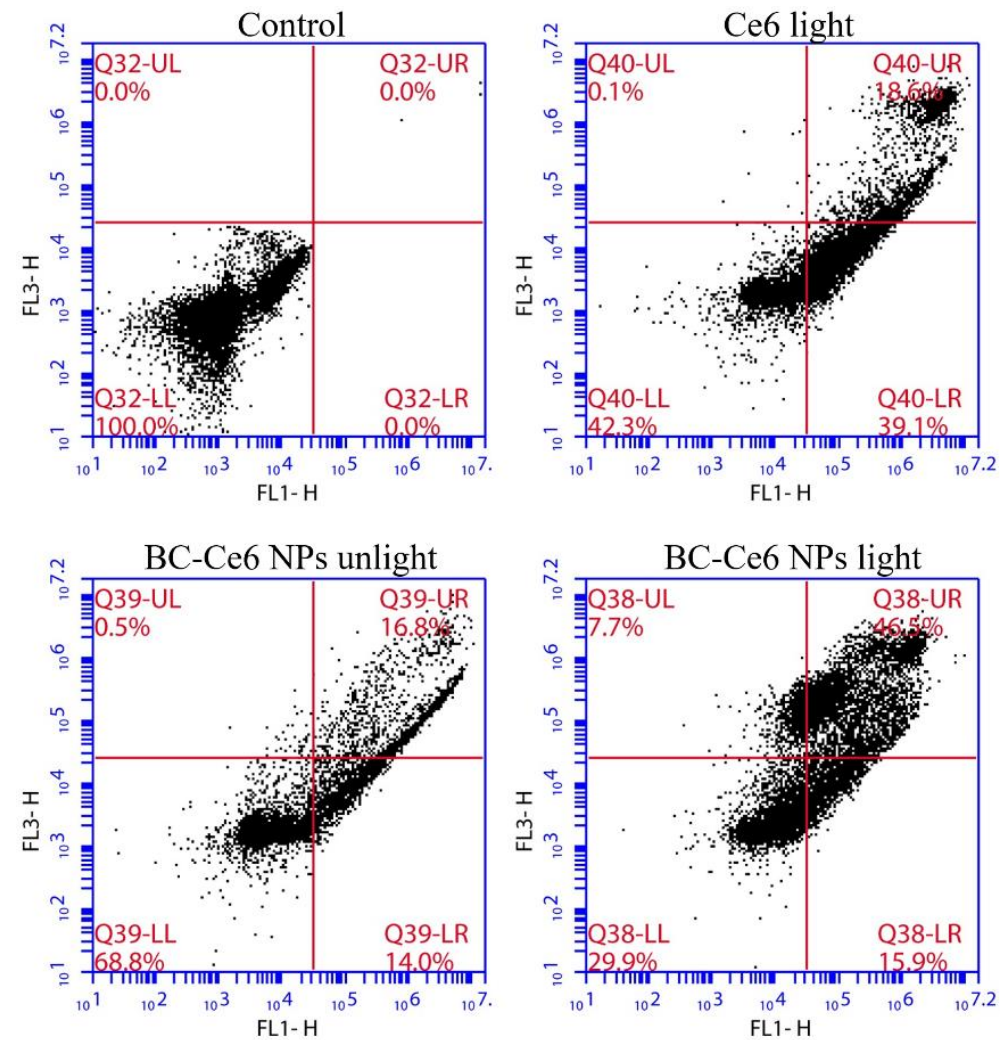

Figure S31. Annexin V-FITC/PI staining for 4T1 cell apoptosis after incubation of free Ce6 or BC-Ce6 NPs (Eq.Ce6: $2 \mu \mathrm{g} / \mathrm{mL}$ ) for $4 \mathrm{~h}$, and subsequently irradiation for $10 \mathrm{~min}$ and incubated for additional $10 \mathrm{~h}$, then detected by flow cytometry. 

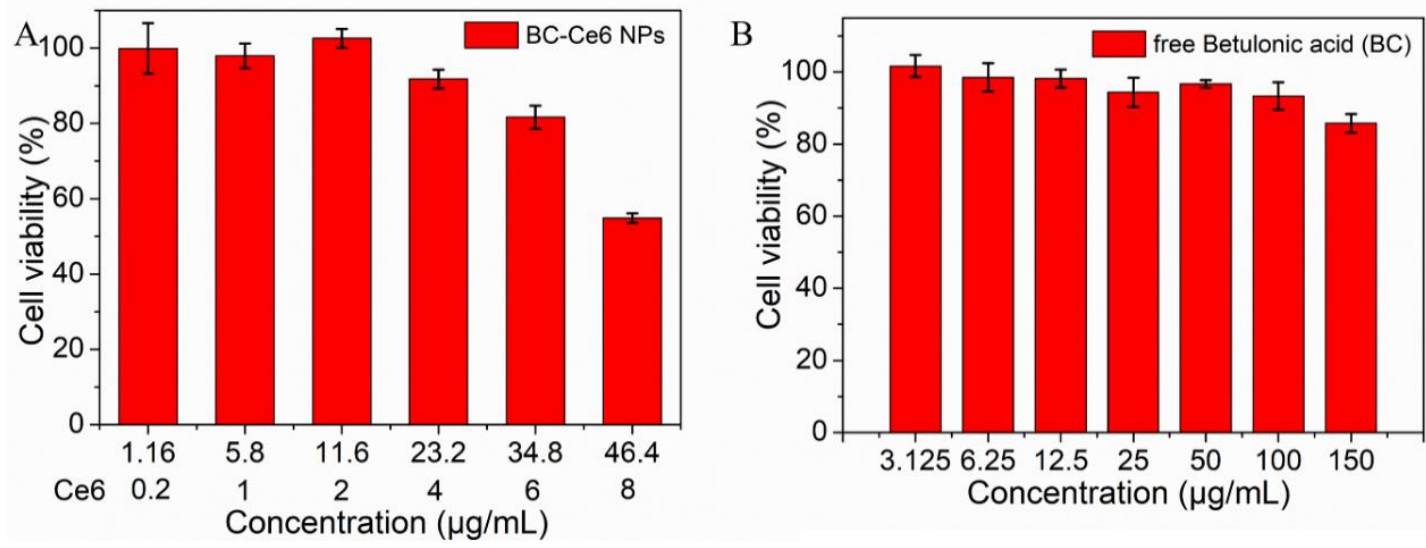

Figure S32. Dark toxicity of (A) BC-Ce6 NPs and (B) free betulonic acid (BC) toward L929 cells in dark for incubation of $24 \mathrm{~h}$ at all indicated concentrations. Admittedly, due to the own excellent anticancer activity of BC and the significant enhanced intracellular internalization of BC-Ce6 NPs, higher concentrations of BC-Ce6 NPs (Eq. Ce6: $8.0 \mu \mathrm{g} / \mathrm{mL}$ ) exhibit certain cytotoxicity to L929 cells, yet free BC still have lower dark toxicity even at higher dose of 150 $\mu \mathrm{g} / \mathrm{mL}$, indicating the NPs are biocompatible.

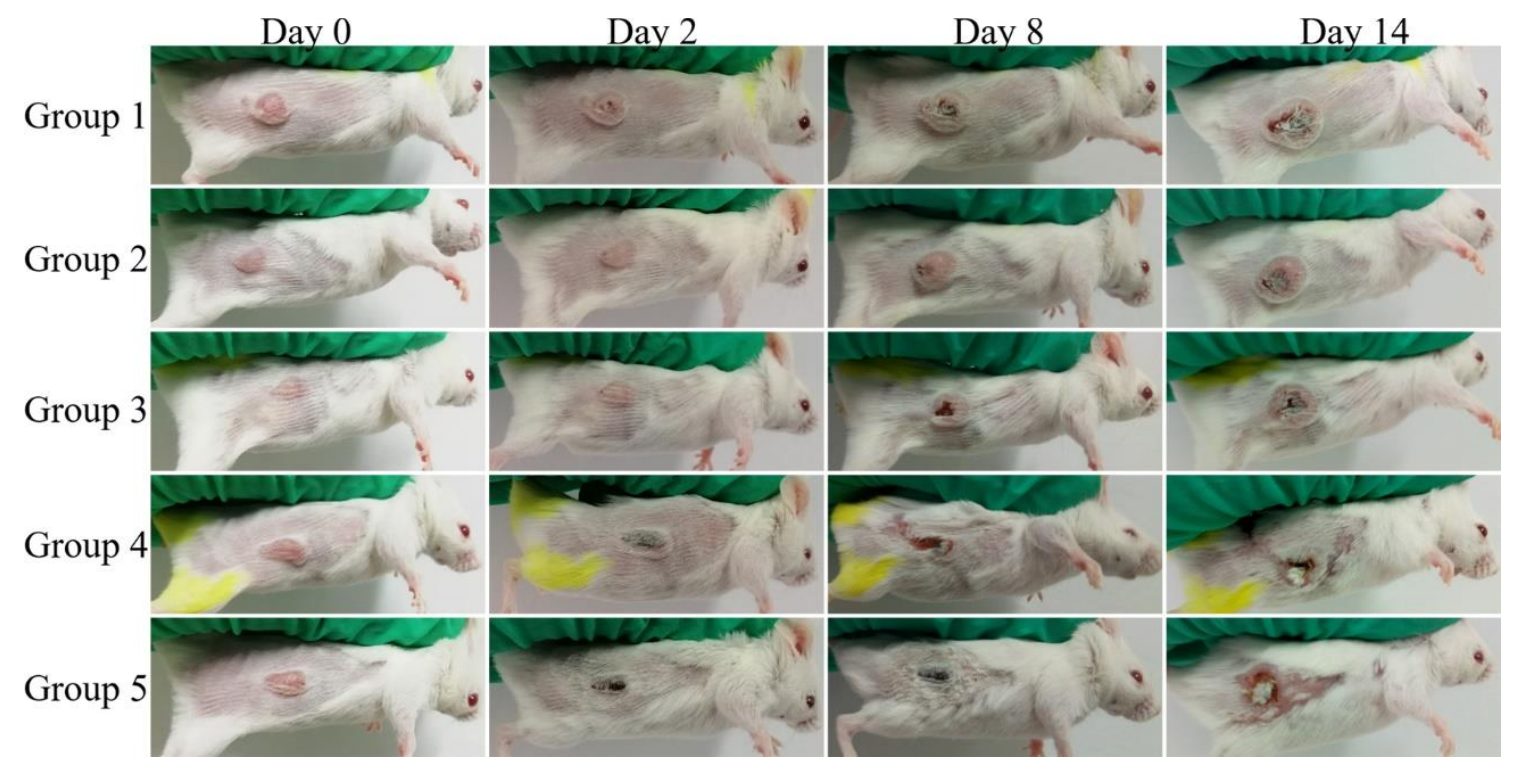

Figure S33. Representative mice photographs before treatment (Day 0) and after therapy for 2, 8 and 14 days in different groups: Group 1) Control; 2) free BC; 3) BC-Ce6 NPs without irradiation; 4) free Ce6 with irradiation; 5) BC-Ce6 NPs with irradiation, respectively. 


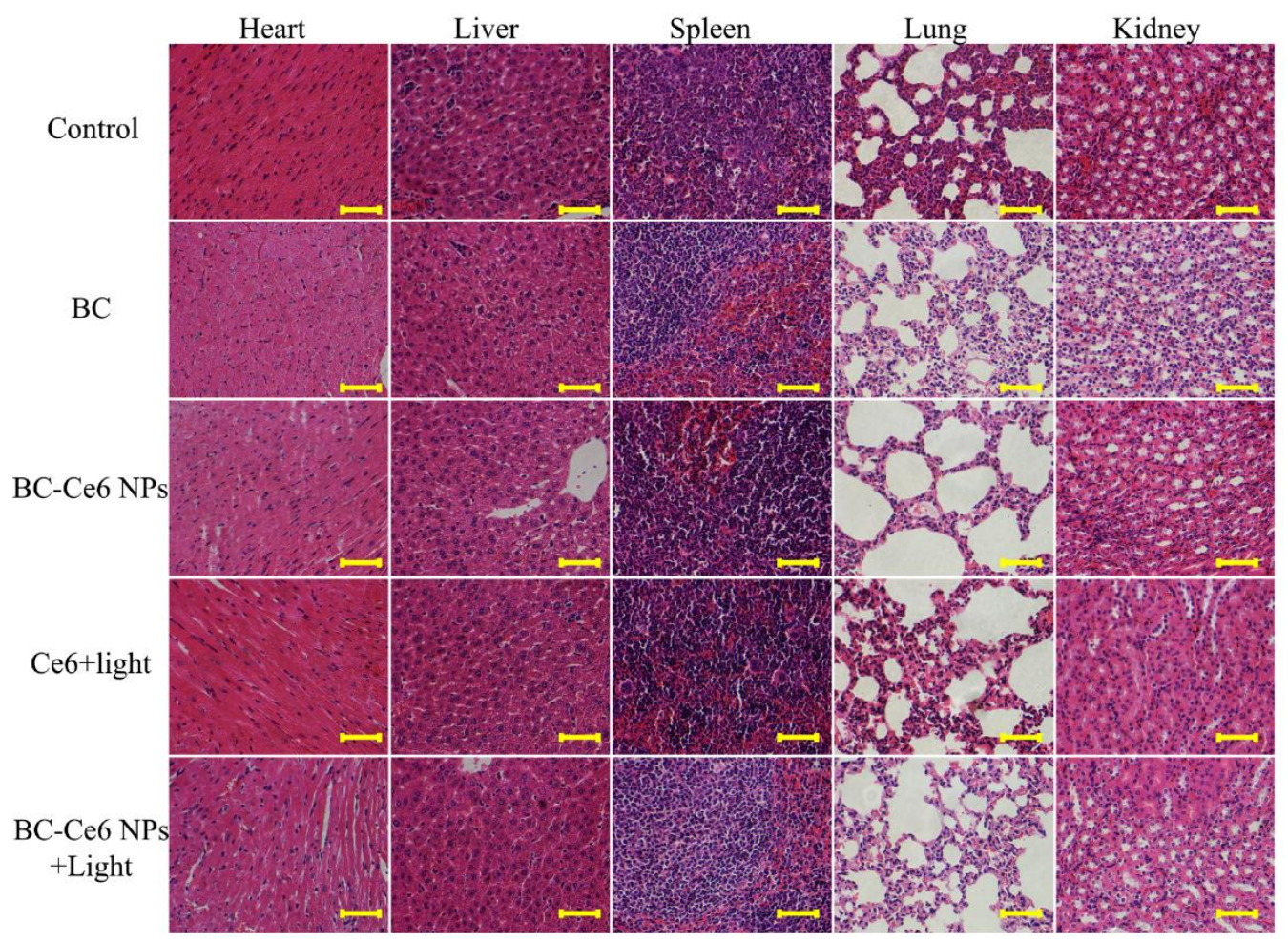

Figure S34. H\&E staining of major organs including heart, liver, spleen, lung, kidney obtained after 14 days treatment in different groups as indicated. Scale bar: $20 \mu \mathrm{m}$.
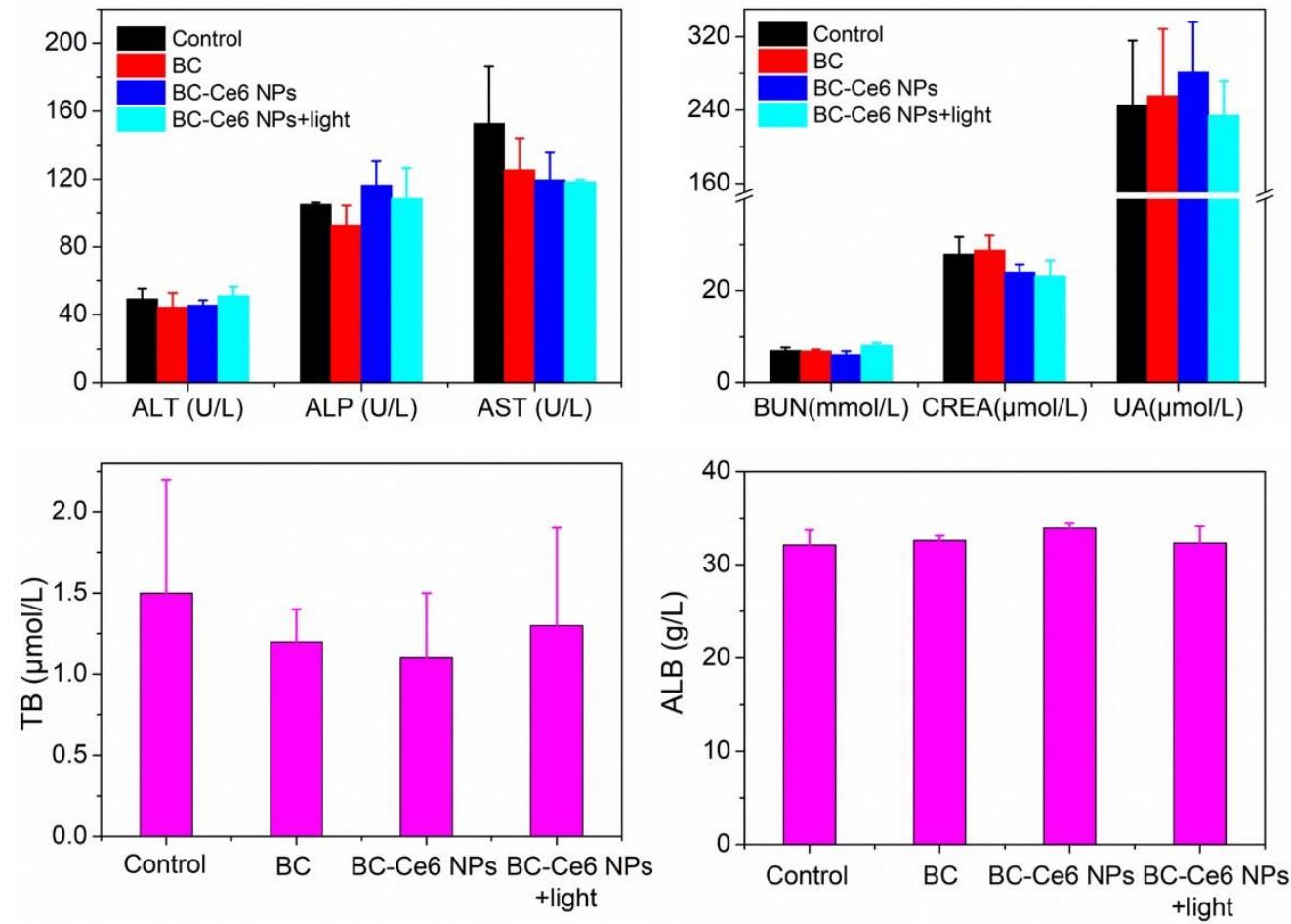

Figure S35.The main liver function markers (ALT, ALP, AST), renal function markers (BUN, CREA, UA), and other biochemical parameters (ALB, TB) in serum biochemistry analysis of mice at the end of treatment, compared with normal mice (control group). 


\section{References}

[1] Yang, X.; Ma, C.; Chen, Z.; Liu, J.; Liu, F.; Xie, R.; Zhao, H.; Deng, G.; Chen, A. T.; Gong, N.; Yao, L.; Zuo, P.; Zhi, K.; Wang, J.; Gao, X.; Wang, J.; Fan, L.; Zhou, J., Single small molecule-assembled nanoparticles mediate efficient oral drug delivery. Nano Research 2019, 12, 2468-2476.

[2] 2. Zhi, K.; Wang, J.; Zhao, H.; Yang, X., Self-assembled small molecule natural product gel for drug delivery: A breakthrough in new application of small molecule natural products. Acta. Pharm. Sin. B. 2020, 10, 913.

[3] 3. Lv, Z.; Wei, H.; Li, Q.; Su, X.; Liu, S.; Zhang, K. Y.; Lv, W.; Zhao, Q.; Li, X.; Huang, W., Achieving efficient photodynamic therapy under both normoxia and hypoxia using cyclometalated $\mathrm{Ru}$ (II) photosensitizer through type I photochemical process. Chem. Sci. 2018, 9, 502-512.

[4] 4. Ouyang, J.; Wang, L.; Chen, W.; Zeng, K.; Han, Y.; Xu, Y.; Xu, Q.; Deng, L.; Liu, Y.-N., Biomimetic nanothylakoids for efficient imaging-guided photodynamic therapy for cancer. Chem. Commun. 2018, 54, 3468-3471.

[5] 5. Ji, C.; Gao, Q.; Dong, X.; Yin, W.; Gu, Z.; Gan, Z.; Zhao, Y.; Yin, M., A Size-Reducible Nanodrug with an Aggregation-Enhanced Photodynamic Effect for Deep ChemoPhotodynamic Therapy. Angew. Chem. Int. Ed. 2018, 57, 11384-11388.

[6] 6. Zhao, H.; Kalivendi, S.; Zhang, H.; Joseph, J.; Nithipatikom, K.; Vásquez-Vivar, J.; Kalyanaraman, B., Superoxide reacts with hydroethidine but forms a fluorescent product that is distinctly different from ethidium: potential implications in intracellular fluorescence detection of superoxide. Free Radical Biol. Med. 2003, 34, 1359-1368.

[7] 7. Priya Arjunan, K.; Morss Clyne, A., Hydroxyl Radical and Hydrogen Peroxide are Primarily Responsible for Dielectric Barrier Discharge Plasma-Induced Angiogenesis. Plasma Process. Polym. 2011, 8, 1154-1164.

[8] Gaussian 09, Revision E.01, M. J. Frisch, G. W. Trucks, H. B. Schlegel, G. E. Scuseria, M. A. Robb, J. R. Cheeseman, G. Scalmani, V. Barone, B. Mennucci, G. A. Petersson, H. Nakatsuji, M. Caricato, X. Li, H. P. Hratchian, A. F. Izmaylov, J. Bloino, G. Zheng, J. L. Sonnenberg, M. Hada, M. Ehara, K. Toyota, R. Fukuda, J. Hasegawa, M. Ishida, T. Nakajima, Y. Honda, O. Kitao, H. Nakai, T. Vreven, J. A. Montgomery, Jr., J. E. Peralta, F. Ogliaro, M. Bearpark, J. J. Heyd, E. Brothers, K. N. Kudin, V. N. Staroverov, T. Keith, R. Kobayashi, J. Normand, K. Raghavachari, A. Rendell, J. C. Burant, S. S. Iyengar, J. Tomasi, M. Cossi, N. Rega, J. M. Millam, M. Klene, J. E. Knox, J. B. Cross, V. Bakken, C. Adamo, J. Jaramillo, R. Gomperts, R. E. Stratmann, O. Yazyev, A. J. Austin, R. Cammi, C. Pomelli, J. W. Ochterski, R. L. Martin, K. Morokuma, V. G. Zakrzewski, G. A. Voth, P. Salvador, J. J. Dannenberg, S. Dapprich, A. D. Daniels, O. Farkas, J. B. Foresman, J. V. Ortiz, J. Cioslowski, and D. J. Fox, Gaussian, Inc., Wallingford CT, 2013.

[9] Liu, K.; Kang, Y.; Ma, G.; Möhwald, H.; Yan, X., Molecular and mesoscale mechanism for hierarchical self-assembly of dipeptide and porphyrin light-harvesting system. Phys. Chem. Chem. Phys. 2016, 18, 16738-16747.

[10]Zhang, F.; Ni, Q.; Jacobson, O.; Cheng, S.; Liao, A.; Wang, Z.; He, Z.; Yu, G.; Song, J.; Ma, Y.; Niu, G.; Zhang, L.; Zhu, G.; Chen, X., Polymeric Nanoparticles with a GlutathioneSensitive Heterodimeric Multifunctional Prodrug for In Vivo Drug Monitoring and Synergistic Cancer Therapy. Angew. Chem. Int. Ed. 2018, 57, 7066-7070.

[11]Cheng, J.; Tan, G.; Li, W.; Zhang, H.; Wu, X.; Wang, Z.; Jin, Y., Facile synthesis of chitosan assisted multifunctional magnetic Fe3O4@SiO2@CS@pyropheophorbide-a fluorescent nanoparticles for photodynamic therapy. New J. Chem. 2016, 40, 8522-8534.

[12]Li, Z.; Hu, Y.; Howard, K. A.; Jiang, T.; Fan, X.; Miao, Z.; Sun, Y.; Besenbacher, F.; Yu, M., Multifunctional Bismuth Selenide Nanocomposites for Antitumor ThermoChemotherapy and Imaging. ACS Nano 2015, 10, 984-997. 
[13]Liang, R.; Tian, R.; Ma, L.; Zhang, L.; Hu, Y.; Wang, J.; Wei, M.; Yan, D.; Evans, D. G.; Duan, X., A supermolecular photosensitizer with excellent anticancer performance in photodynamic therapy. Adv. Funct. Mater. 2014, 24, 3144-3151.

[14] Kadish, K. M.; Smith, K. M.; Guilard, R., Handbook of Porphyrin Science (Volumes 610): With Applications to Chemistry, Physics, Materials Science, Engineering, Biology and Medicine. World Scientific: 2010; Vol. 2. 